ESAIM: M2AN 48 (2014) 1529-1555

DOI: $10.1051 / \mathrm{m} 2 \mathrm{an} / 2014008$
ESAIM: Mathematical Modelling and Numerical Analysis

www.esaim-m2an.org

\title{
MATHEMATICAL MODELING OF TIME-HARMONIC AEROACOUSTICS WITH A GENERALIZED IMPEDANCE BOUNDARY CONDITION
}

\author{
ERIC LUNEVILlE ${ }^{1}$ AND JEAN-FrANCOIS MERCIER ${ }^{1}$
}

\begin{abstract}
We study the time-harmonic acoustic scattering in a duct in presence of a flow and of a discontinuous impedance boundary condition. Unlike a continuous impedance, a discontinuous one leads to still open modeling questions, as in particular the singularity of the solution at the abrupt transition and the choice of the right unknown to formulate the scattering problem. To address these questions we propose a mathematical approach based on variational formulations set in weighted Sobolev spaces. Considering the discontinuous impedance as the limit of a continuous boundary condition, we prove that only the problem formulated in terms of the velocity potential converges to a well-posed problem. Moreover we identify the limit problem and determine some Kutta-like condition satisfied by the velocity: its convective derivative must vanish at the ends of the impedance area. Finally we justify why it is not possible to define limit problems for the pressure and the displacement. Numerical examples illustrate the convergence process.
\end{abstract}

Mathematics Subject Classification. 35J20, 35J05.

Received December 3, 2012. Revised May 18, 2013.

Published online August 13, 2014.

\section{INTRODUCTION}

We are interested in the modeling of sound propagation in lined ducts with flow. This problem has been extensively studied, in particular because of its industrial applications, especially acoustic treatment used on the inside surface of commercial aircraft jet engines for fan noise reduction. We consider a 2D straight duct, a uniform subsonic mean flow with lining of impedance type, described by the Ingard-Myers boundary condition $[1,2]$. This condition incorporates both the impedance of the lining and the effect of the slipping mean flow $[3,4]$.

In this paper we focus on the case of sound scattering by liner discontinuities. We consider a localized lining where the treated area is a finite segment and involving two impedance discontinuities between a hard wall and a lined wall. Due to the flow such sharp transition leads to modeling and mathematical difficulties and there is as of yet no complete description of the physical mechanisms which take place at the transition. Indeed the Myers boundary condition involves a second order tangential derivative: it requires some regularity of the acoustic field on the treated boundary which is rather incompatible with a discontinuous impedance. The two main open questions are the following:

\footnotetext{
Keywords and phrases. Aeroacoustics, scattering of sound in flows, treated boundary, Myers condition, finite elements, variational formulations.

1 POEMS, CNRS-INRIA-ENSTA-ParisTech UMR 7231, 828 Boulevard des Maréchaux, 91762 Palaiseau cedex, France. eric.luneville@ensta.fr; jean-francois.mercier@ensta.fr
} 
- the behavior of the solution near a discontinous transition between rigid and lined surfaces,

- the choice of the right unknown used for the representation of the acoustic field.

The second point is linked to the first since the nature of the singularity observed at the liner discontinuity is different depending on whether the acoustic variable is pressure, velocity potential, displacement potential, etc ...

These issues have been already addressed in previous studies of the acoustic scattering at a liner discontinuity using modal matching methods or Wiener-Hopf approaches, and some insights have been gained. Modematching techniques are widely used, particularly in acoustical engineering, to predict sound propagation in ducts. They require understanding the behavior of the acoustic field at the liner discontinuity since the choice of a particular mode-matching method relates to such a behavior: using pressure and velocity in the standard matching conditions implies that these quantities are sufficiently well behaved to apply the matching. With no flow, mode-matching methods are relatively well established, and the continuity of pressure and axial velocity is generally applied. In lined ducts with flow, the same continuity conditions are currently used in most mode-matching schemes, although the validity of such an approach is questionable since the behavior of the solution at the transition between a hard wall and a lined wall is not well understood. Even when the flow in the duct is uniform, singularities of acoustic pressure can occur at impedance discontinuities and must be taken into account when solutions are matched [5,6]. Different mode-matching conditions, mixing the velocity and the pressure, have been proposed $[5,7-9]$ but there is no rigorous mathematical theory yet. Recently, in order to deal more accurately with liner discontinuity with flow, a modified mode-matching scheme based on conservation of mass and momentum has been proposed to derive the corresponding matching conditions [6]. Different matching conditions have been compared and significant differences have been observed. In the process of deriving the matching conditions, it appeared that in addition to the matching conditions, edge conditions have to be introduced to specify the behavior of the solution at the liner discontinuity. To express such edge conditions, the choice of variable becomes important: the normal acoustic displacement and its axial derivative can be taken to be continuous and such conditions lead to an acoustic field that corresponds to smooth streamlines along the wall. Continuity of the velocity potential can also be considered. This edge condition is relevant when comparing with finite element models based on the full potential theory which is written for the velocity potential.

An alternative way to solve the problem of sound scattering by an impedance discontinuity in a duct with flow is to use Wiener-Hopf techniques [10]. An explicit Wiener-Hopf solution has been derived to describe the scattering of duct modes at a hard-soft wall impedance transition in a circular duct with uniform mean flow $[11,12]$. In Wiener-Hopf techniques, vortex shedding from the wall discontinuity can be taken into account. Such vorticies are due to the excitation of an unstable surface wave [13-15]. When taking into account instability, it is possible to control the behavior of the solution near the discontinuity by introducing a parameter that can be interpreted as the amount of vorticity shedding across the discontinuity. This parameter is fixed by application of the Kutta condition [16,17]. Note that this additional degree of freedom does not appear so clearly in mode-matching techniques but has certainly a link with the edge conditions of the mode-matching techniques. With no Kutta condition a plausible edge condition at $x=0$ (the location of the discontinuity) requires at least a continuous wall streamline $r=1+h(x, t)$ (for a duct of radius $R=1$ ) no more singular than $h=O\left(x^{1 / 2}\right)$ (this implies the same singularity for the pressure $p$ which varies like $h$ on the boundary). When vortex shedding is taken into account, the edge condition requires the wall streamline to be no more singular than $h=O\left(x^{3 / 2}\right)$. The physical relevance of this Kutta condition is still an open question, but the use of a Kutta or no-Kutta condition at the discontinuity has been shown to affect significantly the modal scattering. The available experiments $[18,19]$ give indirect but convincing arguments for the possible existence of instability waves along the liner.

As there is no model to deal completely satisfactorily with a discontinuous transition treated/rigid boundary, we propose as a first step, a complete theoretical analysis for a regularized transition (with a transition area of width $\varepsilon$ ). In a second step, we study the limit process as $\varepsilon$ goes to 0 . A variational approach is chosen, because it enables a rigorous mathematical treatment of the difficulties and also it simplifies the treatment of the regularity at the discontinuous transition: the singular behavior of the solution is naturally controlled by 
the choice of the variational space. No specific treatment like the edge conditions for mode-matching methods or the Kutta condition for the Wiener-Hopf approach has to be introduced. Due to the presence of a second order tangential derivative in the Myers condition, the case of a discontinuous transition is incompatible with a variational approach: the integration by parts of the Myers condition induces some non-controlled boundary terms. Treatment of these terms is delicate. Either it is not precised [20] or else it is achieved thanks to a specific treatment of the pressure gradient [21]: a gradient elimination which requires adding new functions to the finite element basis or the gradient evaluation which imposes to use $C^{1}$ continuous elements. Note that these boundary terms cannot be simply removed since the singular behavior of the solution at the liner discontinuities is unknown.

To avoid such singular boundary terms we consider a smooth transition over a finite distance of length $\epsilon$. Then we take the limit as $\epsilon \rightarrow 0$ to model an abrupt discontinuity. Such an approach has been successfully used to establish appropriate matching conditions [6] or to derive the variational formulation of the potential problem, when both the base flow velocity and the acoustic velocity derive from scalar potentials [22]. We propose a complete theoretical analysis for such a regularized transition, considering three different unknows: the pressure $p_{\epsilon}$, the velocity potential $\varphi_{\epsilon}$ and the displacement potential $\zeta_{\epsilon}$. Since these unknows are linked by the relations $p_{\epsilon}=D \varphi_{\epsilon}$ and $\varphi_{\epsilon}=D \zeta_{\epsilon}$ where $D$ is the convective derivative, each of them has a different singular behavior at the liner discontinuity. In this paper we will show that the advantages of considering a smooth transition are the following:

(1) the three unknowns, $p_{\epsilon}$ or $\varphi_{\epsilon}$ or $\zeta_{\epsilon}$ satisfy different variational formulations which are all well-posed, considering weighted sobolev spaces [23,24],

(2) no boundary condition at the liner ends are necessary to derive any of the variational formulations.

The limit $\epsilon \rightarrow 0$ is delicate to perform since we have to face a singularly perturbed problem [25-28]: the solution for $\epsilon=0$ is more regular on the treated boundary than the solution for any finite value of $\epsilon$. This sudden change of variational space has to be treated carefully. In the following we will prove that

- we can define a limit problem (for $\epsilon=0$ ) when considering the velocity potential formulation and that $\varphi_{\epsilon}$ converges to the solution $\varphi_{0}$ of the limit problem when $\epsilon \rightarrow 0$,

- $\varphi_{0}$ satisfies a Kutta-like condition at the liner ends: $D \varphi_{0}=0$

- only the velocity formulation converges to a well-posed variational problem when $\epsilon \rightarrow 0$,

- we can formally determine the limit of $p_{\epsilon}$ and of $\zeta_{\epsilon}$ and understand why they don't satisfy a well-posed variational limit problem,

- the limit $p_{0}$ of $p_{\epsilon}$ is discontinuous on the wall and vanishes at the end of the treated area.

The outline of the paper is the following. In Section 2 we present the equations for the three unknowns-velocity potential, pressure and displacement-and we explain the difficulties due to discontinuous impedance. Sections 3 and 4 focus on the velocity potential formulation. In Section 3 the well-posedness of the velocity potential formulation for a continuous boundary is proved, introducing weighted Sobolev spaces. Section 4 concerns the convergence of the regularized velocity potential formulation to the limit problem when $\varepsilon$ goes to 0 . The variational formulations for the two other unknowns are presented in Section 5 and the limit pressure and limit displacement are exhibited. Finally, Section 6 is concerned with the numerical illustrations of the limit process. We have reported in the appendix the intricate mathematical proofs.

\section{Problem Setting}

\subsection{Geometry and equations}

We consider a two-dimensional infinite duct $\tilde{\Omega}_{\infty}=\{(x, y) ; 0<y<H\}$ of height $H$ and of boundary $\partial \tilde{\Omega}_{\infty}=\tilde{\Gamma} \cup \tilde{\Gamma}_{0}^{\infty}$ where $\tilde{\Gamma}=\{(x, y) ; y=H$ and $0<x<L\}$ is the treated boundary and $\tilde{\Gamma}_{0}^{\infty}$ is a hard wall. The 


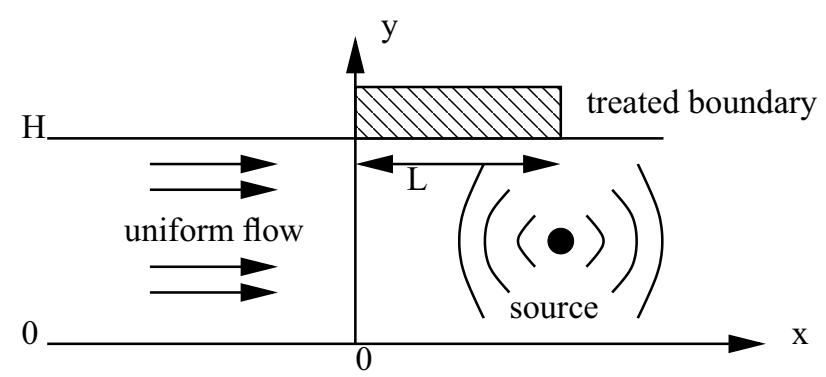

Figure 1. Geometry of the problem.

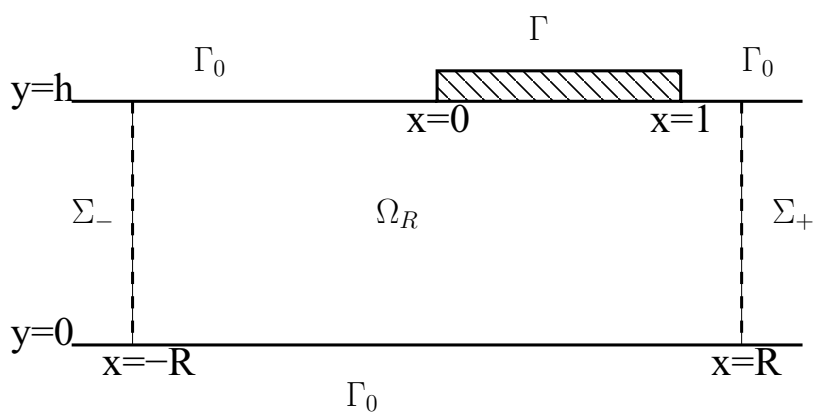

FiguRE 2. Restriction to a bounded problem.

duct is filled with a compressible fluid in a uniform flow of velocity noted $U(U \geq 0)$, see Figure 1 . In the timeharmonic regime and with a time dependence $\mathrm{e}^{-i \omega t}$ omitted, the equation of mass conservation combined with the equation of state, and the equation of momentum conservation are

$$
\left\{\begin{aligned}
\operatorname{div} \boldsymbol{v} & =\frac{1}{\rho_{0} c_{0}^{2}}\left(U \frac{\partial}{\partial x}-i \omega\right) p, \\
\rho_{0}\left(U \frac{\partial}{\partial x}-i \omega\right) \boldsymbol{v} & =-\nabla p
\end{aligned}\right.
$$

where $\boldsymbol{v}$ is the velocity perturbation, $p$ is the acoustic pressure, and $\rho_{0}$ and $c_{0}$ are the constant ambient density and speed of sound in air. Pressure, velocities, and lengths are, respectively, divided by $\rho_{0} c_{0}^{2}, c_{0}$ and $L$ to reduce equations (2.1) to the dimensionless form

$$
\left\{\begin{aligned}
\operatorname{div} \boldsymbol{v} & =D p \\
D \boldsymbol{v} & =-\nabla p
\end{aligned}\right.
$$

where $D=M \partial / \partial x-i k$ is the dimensionless convective derivative with $M=U / c_{0}$ the Mach number and $k=\omega L / c_{0}$ the dimensionless wave number. Eliminating the pressure in equation (2.2) leads to the convected Helmholtz equation

$$
-\Delta p+D^{2} p=0 \text { in } \Omega_{\infty},
$$

where $\Omega_{\infty}=\{(x, y) ; 0<y<h\}$ with $h=H / L$. The boundary condition on the hard walls $\Gamma_{0}$ is the slip condition which reads $\boldsymbol{v} \cdot \boldsymbol{n}=0=\partial p / \partial n$ where $\boldsymbol{n}$ is the normal vector to the wall. The Myers boundary conditions on the liner $\Gamma=\{(x, y) ; y=h$ and $0<x<1\}$ will be detailed in the next section.

In order to formulate the diffraction problem, we will derive a formulation set in the bounded domain $\Omega=\left\{(x, y) \in \Omega_{\infty} ;-R<x<R, 0<y<h\right\}$ (see Fig. 2) where $R>1$ (such that $\partial \Omega$ includes the liner $\Gamma$ ). 
To do so, we define on the fictitious boundaries $\Sigma_{ \pm}=\{(x, y) ; x= \pm R$ and $0<y<h\}$ the exact radiation conditions

$$
\frac{\partial p}{\partial n}=-T_{ \pm} p, \text { on } \Sigma_{ \pm}
$$

The Dirichet-to-Neumann operators are defined (more completely in Appendix 7) as follows

$$
\begin{aligned}
T_{ \pm}: H^{\frac{1}{2}}\left(\Sigma_{ \pm}\right) & \rightarrow H^{-\frac{1}{2}}\left(\Sigma_{ \pm}\right) \\
p & \rightarrow \mp i \sum_{n \geq 0} \beta_{n}^{ \pm}\left(p, w_{n}\right)_{\Sigma^{ \pm}} w_{n}
\end{aligned}
$$

where $w_{n}(y)$ are the transverse guide modes and $(u, v)_{\Sigma^{ \pm}}=\int_{\Sigma^{ \pm}} u \bar{v} \mathrm{~d} y$

\subsection{Impedance boundary condition}

On the liner $\Gamma$ the Myers boundary condition [2] reads

$$
\beta p=-i \omega \boldsymbol{\xi} \cdot \boldsymbol{n},
$$

where $\beta(x)$ is the liner admittance with $\Re e(\beta)>0$ (to absorb sound) and $\boldsymbol{\xi}$ is the fluid displacement, linked to the velocity by $\boldsymbol{v}=D \boldsymbol{\xi}$. In a dimensionless form this boundary condition becomes

$$
Y p=-i k \boldsymbol{\xi} \cdot \boldsymbol{n}
$$

where $Y(x)=\rho_{0} c_{0} \beta(x)$ is the dimensionless admittance. If $Y$ is extended by 0 outside $\Gamma$ then this condition is valid on the whole upper wall $\left(\boldsymbol{\xi} \cdot \boldsymbol{n}=0\right.$ on $\left.\Gamma_{0}\right)$. The Myers boundary condition naturally mixes the pressure and the displacement. It is usual to express it in terms of only one unknown, the pressure, the velocity potential $\varphi$ $(\boldsymbol{v}=\boldsymbol{\nabla} \varphi)$ or the displacement potential $\zeta(\boldsymbol{\xi}=\boldsymbol{\nabla} \zeta)$. Then the Myers boundary condition takes three different forms

(1) Pressure model

Using $D(\boldsymbol{\xi} \cdot \boldsymbol{n})=\boldsymbol{v} \cdot \boldsymbol{n}$ and $D(\boldsymbol{v} \cdot \boldsymbol{n})=-\frac{\partial p}{\partial n}$ leads to

$$
\frac{\partial p}{\partial n}=\frac{1}{i k} D^{2}(Y p)
$$

Note that since $Y$ depends on $x$ it does not commute with the operator $D$.

(2) Velocity potential model

From $p=-D \varphi$ and $D(\boldsymbol{\xi} \cdot \boldsymbol{n})=\frac{\partial \varphi}{\partial n}$ one gets

$$
\frac{\partial \varphi}{\partial n}=\frac{1}{i k} D(Y D \varphi)
$$

(3) Displacement potential model

The links $\varphi=D \zeta$ and $p=-D^{2} \varphi$ lead to

$$
\frac{\partial \zeta}{\partial n}=\frac{Y}{i k} D^{2} \zeta
$$


Note that although the form of the impedance boundary condition depends on the variable choice, this is not the case for the convected Helmholtz equation (2.3): this equation is valid for the pressure, the velocity or the displacement potential.

\subsection{The difficulties of a discontinuous transition}

In this part we will show that whichever is the chosen unknown, $p, \varphi$ or $\zeta$, a discontinuous boundary condition leads to mathematical problems. Indeed for a discontinuous transition due to an admittance constant by parts $Y(x)=Y_{0} \eta(x)$ with $\eta=1$ for $x \in[0,1], \eta=0$ for $x<0$ and $x>1$ and $Y_{0}$ a complex constant, the boundary condition at $y=h$ reads (formally):

(1) for the pressure model

$$
\frac{\partial p}{\partial n}=\frac{Y_{0}}{i k}\left[\left(\delta_{0}^{\prime}-\delta_{1}^{\prime}\right) M^{2} p+2\left(\delta_{0}-\delta_{1}\right) M D p+\eta D^{2} p\right] ;
$$

(2) for the velocity potential model

$$
\frac{\partial \varphi}{\partial n}=\frac{Y_{0}}{i k}\left[\left(\delta_{0}-\delta_{1}\right) M D \varphi+\eta D^{2} \varphi\right]
$$

(3) for the displacement potential model

$$
\frac{\partial \zeta}{\partial n}=\frac{Y_{0}}{i k} \eta D^{2} \zeta
$$

For the pressure and the velocity potential models the boundary conditions involve Dirac distributions and thus it requires some appropriate regularity properties for the pressure and for the velocity potential at the liner edges (more regular than $H^{1}(\Omega)$ ). The displacement potential seems to be the right unknown to choose since no Dirac distribution appears in the boundary condition. However when establishing the variational formulation of the scattering problem, the integration by parts introduces some non-conventional terms on the wall at $y=h$

$$
\frac{Y_{0}}{i k} \int_{\mathbb{R}} \eta\left(D^{2} \zeta\right) \bar{\zeta} \mathrm{d} x=\frac{Y_{0}}{i k} \int_{\Gamma}\left(D^{2} \zeta\right) \bar{\zeta} \mathrm{d} x=-\frac{Y_{0}}{i k}\left\{\int_{\Gamma} D \zeta \overline{D \zeta} \mathrm{d} x-[M(D \zeta) \bar{\zeta}]_{x=0}^{x=1}\right\}
$$

The term $[(D \zeta) \bar{\zeta}]_{x=0}^{x=1}$ is not compliant with a variational approach, unless $D \zeta=0$ or $\zeta=0$ at $x=0$ and $x=1$ but such an assumption requires knowing the behavior of $\zeta$ at the liner edges.

An alternative to treat a discontinuous boundary condition is to consider a smooth impedance transition. Then the three unknowns lead to well-posed variational formulations, which gives a mathematical framework to study the convergence toward a discontinuous impedance. In particular we will prove that the velocity potential converges to a limit problem whose solution $\varphi$ satisfies on the treated part $\Gamma: \varphi \in H^{1}(\Gamma), \partial \varphi / \partial \boldsymbol{n}=$ $(1 / i k) D(Y D \varphi)$ in $\mathcal{D}^{\prime}(\Gamma)$ with $D \varphi\left(0^{+}, h\right)=0=D \varphi\left(1^{-}, h\right)$. The last boundary conditions at the ends of the treated wall was not obvious.

Remark 2.1. Since the admittance is obtained by some homogenization process completely neglecting the transition zone from the lined to the rigid part of the walls, an abrupt change in the admittance is physically not relevant and a smooth transition is certainly closer to reality. However, the explicit expression of this smooth transition is unknown, and it is useful to determine the limit problem without continuous transition. 


\section{VELOCITY SOLUTION IN THE REGULARIZED CASE}

\subsection{Variational formulation}

In this section we restrict ourselves to a regularized impedance boundary condition and we focus on the velocity potential problem for a source $f \in L^{2}(\Omega)$ compactly supported in $\Omega$

$$
\begin{cases}-\Delta \varphi+D^{2} \varphi=f, & \text { in } \Omega, \\ \frac{\partial \varphi}{\partial n}=0, & \text { on } \Gamma_{0}, \\ \frac{\partial \varphi}{\partial n}=-T_{ \pm} \varphi, & \text { on } \Sigma^{ \pm} \\ \frac{\partial \varphi}{\partial n}=\frac{Y_{0}}{i k} D\left(\eta_{\varepsilon} D \varphi\right), & \text { on } \Gamma .\end{cases}
$$

$Y_{0} \in \mathbb{C}$ is a constant and $\eta_{\varepsilon}(x) \in C^{0}(\mathbb{R})$ is the regularisation function which for $\varepsilon \leq 1 / 2$ is chosen as

$$
\eta_{\varepsilon}=\left\{\begin{array}{cl}
\frac{x}{\varepsilon} \quad \text { if } x \leq \varepsilon \\
1 \quad \text { if } \varepsilon \leq x \leq 1-\varepsilon, \\
\frac{1-x}{\varepsilon} \quad \text { if } 1-\varepsilon \leq x \leq 1 .
\end{array}\right.
$$

Let us introduce the two weighted Sobolev spaces

$$
V_{\varepsilon}=\left\{\varphi \in H^{1}(\Omega), \eta_{\varepsilon}^{\frac{1}{2}} D\left(\left.\varphi\right|_{\Gamma}\right) \in L^{2}(\Gamma)\right\}
$$

and

$$
V=\left\{\varphi \in H^{1}(\Omega), \eta^{\frac{1}{2}} D\left(\left.\varphi\right|_{\Gamma}\right) \in L^{2}(\Gamma)\right\}
$$

where $D\left(\left.\varphi\right|_{\Gamma}\right)$ has to be understood in the distributional sense on $\Gamma$ and where we note

$$
\eta=\frac{1}{2} \eta_{\frac{1}{2}}=\left\{\begin{array}{cl}
x & \text { if } x \leq \frac{1}{2}, \\
1-x & \text { if } \frac{1}{2} \leq x \leq 1 .
\end{array}\right.
$$

Note that $\left\{\varphi \in H^{1}(\Omega),\left.\varphi\right|_{\Gamma} \in H^{1}(\Gamma)\right\} \subset V \subset\left\{\varphi \in H^{1}(\Omega),\left.\varphi\right|_{\Gamma} \in H_{\mathrm{loc}}^{1}(\Gamma)\right\}$.

Problem (3.1) has the equivalent variational form

$$
\left\{\begin{array}{l}
\text { Find } \varphi_{\varepsilon} \in V \text { such that } \\
a_{\varepsilon}\left(\varphi_{\varepsilon}, \psi\right)=\int_{\Omega} f \bar{\psi} \mathrm{d} x \mathrm{~d} y \text { for all } \psi \in V,
\end{array}\right.
$$

where the sesquilinear form $a_{\varepsilon}(\varphi, \psi)$ is defined by

$$
a_{\varepsilon}(\varphi, \psi)=\int_{\Omega}(\nabla \varphi \cdot \nabla \bar{\psi}-D \varphi \overline{D \psi}) \mathrm{d} x \mathrm{~d} y+\left\langle S_{ \pm} \varphi, \psi\right\rangle_{ \pm}+\frac{Y_{0}}{i k} \int_{\Gamma} \eta_{\varepsilon} D \varphi \overline{D \psi} \mathrm{d} x .
$$

The Dirichlet-to-Neumann operators $S^{ \pm}$are defined by

$$
S_{ \pm} \varphi=\left(1-M^{2}\right) T_{ \pm} \varphi \mp i k M \varphi=-i \sum_{n \geq 0} \sqrt{k^{2}-\left(\frac{n \pi}{h}\right)^{2}\left(1-M^{2}\right)}\left(\varphi, w_{n}\right)_{\Sigma^{ \pm}} w_{n},
$$

and where $\langle\cdot, \cdot\rangle_{ \pm}$is the duality product between $H^{\frac{1}{2}}\left(\Sigma^{ \pm}\right)$and its dual $H^{-\frac{1}{2}}\left(\Sigma^{ \pm}\right)$. 
Remark 3.1. Note that the natural space should be $V_{\varepsilon}$ defined in (3.3) instead of $V$. It is easy to see that for all $0<\varepsilon \leq 1 / 2$, the spaces family $V_{\varepsilon}$ does not depend on $\varepsilon$ and more precisely that all the spaces $V_{\varepsilon}$ are equal to $V$. Indeed $V_{\varepsilon}$ is the space of the functions $\varphi \in H^{1}(\Omega)$ with $\left.\varphi\right|_{\Gamma} \in H_{\mathrm{loc}}^{1}$ such that $x|\partial \varphi(x, h) / \partial x|^{2}$ is integrable at $x=0$ and $(1-x)|\partial \varphi(x, h) / \partial x|^{2}$ is integrable at $x=1$.

Note also that the boundary term obtained after integration by parts

$$
\frac{Y_{0}}{i k} M\left[\eta_{\varepsilon}(D \varphi) \bar{\psi}\right]_{x=0}^{x=1}
$$

vanishes thanks to $\eta_{\varepsilon}(0)=0=\eta_{\varepsilon}(1)$ without requiring any condition on $\psi$.

Remark 3.2. In fact the space $V$ controls the singular behavior of the velocity potential at the discontinuous transition: the solution is such that $\varphi \in H^{1}(\Omega)$ and $\left.\varphi\right|_{\Gamma} \in H_{\mathrm{loc}}^{1}(\Gamma)$ and as we will show later (Lem. A.2) we have at the liner ends the regularity $\lim _{x \rightarrow 0^{+}} x^{\frac{1}{2}} \varphi(x, h)=0=\lim _{x \rightarrow 1^{-}}(1-x)^{\frac{1}{2}} \varphi(x, h)$. Although the behavior of the solution at the liner ends is not required to pass to the limit $\varepsilon \rightarrow 0$, it is possible to specify more explicitely the nature of the singularities at the liner ends. For instance in the neighborhood of the point $(0, h)$, using polar coordinates $(r, \theta)$ and the Euler change of variable $(r, \theta) \rightarrow(z, \theta)$ with $z=-\log r$, such singular behaviors can be found, analytically if $Y_{0}$ is real, numerically in other cases by solving a dispersion relation.

Our first aim is to prove the well-posedness of problem (3.1) in $V$, which equipped with the norm $\|\varphi\|_{V}^{2}=$ $\|\varphi\|_{H^{1}(\Omega)}^{2}+\left\|\eta^{\frac{1}{2}} D\left(\left.\varphi\right|_{\Gamma}\right)\right\|_{L^{2}(\Gamma)}^{2}$ is an Hilbert space.

\subsection{Well-posedness of the regularized problem}

To prove the well-posedness we mainly follow a rather standard procedure, for instance detailed in [29]. However the presence of an impedance boundary condition introduces new difficulties and requires the introduction of new proof arguments, which we will detail now.

We now establish a Fredholm decomposition of formulation $\left(P_{\varepsilon}\right)$. It is obvious that

$$
a_{\varepsilon}(\varphi, \psi)=b_{\varepsilon}(\varphi, \psi)+c_{\varepsilon}(\varphi, \psi),
$$

where

$$
b_{\varepsilon}(\varphi, \psi)=\int_{\Omega}\left[\left(1-M^{2}\right) \frac{\partial \varphi}{\partial x} \frac{\overline{\partial \psi}}{\partial x}+\frac{\partial \varphi}{\partial y} \frac{\partial \psi}{\partial y}+\varphi \bar{\psi}\right] \mathrm{d} x \mathrm{~d} y+\left\langle S_{ \pm}^{e} \varphi, \psi\right\rangle_{ \pm}+\frac{Y_{0}}{i k} \int_{\Gamma} \eta_{\varepsilon} D \varphi \overline{D \psi} \mathrm{d} x
$$

and

$$
c_{\varepsilon}(\varphi, \psi)=\int_{\Omega}\left[-\left(1+k^{2}\right) \varphi \bar{\psi}+i k M\left(\varphi \overline{\frac{\partial \psi}{\partial x}}-\frac{\partial \varphi}{\partial x} \bar{\psi}\right)\right] \mathrm{d} x \mathrm{~d} y+\left\langle S_{ \pm}^{p} \varphi, \bar{\psi}\right\rangle_{ \pm} .
$$

The Dirichlet-to-Neuman operators $S_{ \pm}^{p / e}$ are defined by

$$
\left\{\begin{array}{l}
S_{ \pm}^{p} \varphi=-i \sum_{n \leq N} \sqrt{k^{2}-\left(\frac{n \pi}{h}\right)^{2}\left(1-M^{2}\right)}\left(\varphi, w_{n}\right)_{\Sigma^{ \pm}} w_{n} \\
S_{ \pm}^{e} \varphi=\sum_{n>N} \sqrt{\left(\frac{n \pi}{h}\right)^{2}\left(1-M^{2}\right)-k^{2}}\left(\varphi, w_{n}\right)_{\Sigma^{ \pm}} w_{n}
\end{array}\right.
$$

where $N$ is the integer part of $k h / \pi \sqrt{1-M^{2}}$. $S^{p}$ is the "propagative" part of $S$ in the sense that only the propagative guide modes are taken into account in the modal expansion and $S^{e}$ is the "evanescent" part. 
Theorem 3.3. For $\Re e\left(Y_{0}\right)>0$, problem $\left(P_{\varepsilon}\right)$ is of Fredholm type.

Proof. This theorem results from the following properties:

(1) Coercivity

Taking $\psi=\varphi$ leads to

$$
b_{\varepsilon}(\varphi, \varphi)=\int_{\Omega}\left[\left(1-M^{2}\right)\left|\frac{\partial \varphi}{\partial x}\right|^{2}+\left|\frac{\partial \varphi}{\partial y}\right|^{2}+|\varphi|^{2}\right] \mathrm{d} x \mathrm{~d} y+\left\langle S_{ \pm}^{e} \varphi, \varphi\right\rangle_{ \pm}-\frac{i Y_{0}}{k} \int_{\Gamma} \eta_{\varepsilon}|D \varphi|^{2} \mathrm{~d} x
$$

Let us note $i Y_{0}=i \Re e\left(Y_{0}\right)-\Im m\left(Y_{0}\right)=\left|Y_{0}\right| \mathrm{e}^{\mathrm{i} \theta}$ where $\left.\theta=\operatorname{Arg}\left(Y_{0}\right)+\pi / 2 \in\right] 0, \pi[$. We introduce the decomposition

$$
b_{\varepsilon}(\varphi, \varphi)=\alpha(\varphi)-\mathrm{e}^{\mathrm{i} \theta} \beta(\varphi)+\gamma(\varphi)
$$

where we have introduced the positive forms

$$
\begin{aligned}
& \alpha(\varphi)=\int_{\Omega}\left[\left(1-M^{2}\right)\left|\frac{\partial \varphi}{\partial x}\right|^{2}+\left|\frac{\partial \varphi}{\partial y}\right|^{2}+|\varphi|^{2}\right] \mathrm{d} x \mathrm{~d} y, \\
& \beta(\varphi)=\frac{\left|Y_{0}\right|}{k} \int_{\Gamma} \eta_{\varepsilon}|D \varphi|^{2} \mathrm{~d} x \\
& \gamma(\varphi)=\left\langle S_{ \pm}^{e} \varphi, \varphi\right\rangle_{ \pm}=\sum_{n \geq N} \sqrt{\left(\frac{n \pi}{h}\right)^{2}\left(1-M^{2}\right)-k^{2}}\left|\left(\varphi, w_{n}\right)_{\Sigma^{ \pm}}\right|^{2} .
\end{aligned}
$$

The lower bound: $\left|b_{\varepsilon}(\varphi, \varphi)\right|^{2}=\left|\lambda-\mathrm{e}^{\mathrm{i} \theta} \beta\right|^{2}=(\lambda-\beta)^{2}+4 \lambda \beta \sin ^{2}\left(\frac{\theta}{2}\right) \geq \sin ^{2}\left(\frac{\theta}{2}\right)(\lambda+\beta)^{2}$ for $\lambda=\alpha+\gamma$, leads to the coercivity constant

$$
\left|b_{\varepsilon}(\varphi, \varphi)\right| \geq \sin \left(\frac{\theta}{2}\right) \min \left[\left(1-M^{2}\right), \frac{|Y|}{k}\right]\|\varphi\|_{V}^{2},
$$

since $\gamma(\varphi) \geq 0$ and $\eta_{\varepsilon} \geq \eta$. Note that $\sin (\theta / 2) \neq 0$ because $\theta=0$ implies that $\Re e\left(Y_{0}\right)=0$.

Remark 3.4. In the unphysical case $\Re e\left(Y_{0}\right)<0, b_{\varepsilon}(\varphi, \psi)$ is also coercive. If $\Re e\left(Y_{0}\right)=0$ there is coercivity if $\Im m\left(Y_{0}\right)>0$.

(2) The bounded operator $C_{\varepsilon}$ of $V$ defined by the identity

$$
\left(C_{\varepsilon} \varphi, \psi\right)_{V}=c_{\varepsilon}(\varphi, \psi) \text { for all } \varphi, \psi \in V
$$

is compact. Indeed

$$
\begin{aligned}
c_{\varepsilon}(\varphi, \psi)= & \int_{\Omega}\left[-\left(1+k^{2}\right) \varphi \bar{\psi}+i k M\left(\varphi \overline{\frac{\partial \psi}{\partial x}}-\frac{\partial \varphi}{\partial x} \bar{\psi}\right)\right] \mathrm{d} x \mathrm{~d} y, \\
& -i \sum_{n \leq N} \sqrt{k^{2}-\left(\frac{n \pi}{h}\right)^{2}\left(1-M^{2}\right)}\left(\varphi, w_{n}\right)_{\Sigma^{ \pm}}\left(w_{n}, \psi\right)_{\Sigma^{ \pm}} .
\end{aligned}
$$

Therefore it is infered from the compacity of the embedding of $H^{1}(\Omega)$ (and thus of $V$ ) into $L^{2}(\Omega)$ and from the fact that the number of terms in the sum is finite.

By using the Fredholm alternative, problem $\left(P_{\varepsilon}\right)$ is well-posed if and only if the homogeneous problem

$$
\text { Find } \varphi \in V \text { such that } a_{\varepsilon}(\varphi, \psi)=0 \text { for all } \psi \in V \text {, }
$$

has no solution except the trivial one $\varphi=0$. We first characterize this solution. 
Lemma 3.5. For $\Re e\left(Y_{0}\right)>0$, if $\varphi$ is a nontrivial solution of (3.5), then it is a solution of

$$
\text { Find } \varphi \in H^{1}(\Omega) \text { such that } a^{\prime}(\varphi, \psi)=0 \quad \forall \psi \in H^{1}(\Omega)
$$

where

$$
a^{\prime}(\varphi, \psi)=\int_{\Omega}(\nabla \varphi \cdot \nabla \bar{\psi}-D \varphi \overline{D \psi}) \mathrm{d} x \mathrm{~d} y+\left\langle S_{ \pm}^{e} \varphi, \bar{\psi}\right\rangle_{ \pm} .
$$

Proof. Let us recall first that

$$
a_{\varepsilon}(\varphi, \psi)=a^{\prime}(\varphi, \psi)+\left\langle S_{ \pm}^{p} \varphi, \psi\right\rangle_{ \pm}+\frac{Y_{0}}{i k} \int_{\Gamma} \eta_{\varepsilon} D \varphi \overline{D \psi} \mathrm{d} x
$$

The proof consists in considering $\Im m\left[a_{\varepsilon}(\varphi, \varphi)\right]=0$. It reads

$$
-\Im m\left[a_{\varepsilon}(\varphi, \varphi)\right]=\sum_{n \leq N} \sqrt{k^{2}-\left(\frac{n \pi}{h}\right)^{2}\left(1-M^{2}\right)}\left|\left(\varphi, w_{n}\right)_{\Sigma^{ \pm}}\right|^{2}+\frac{1}{k} \Re e\left(Y_{0}\right) \int_{\Gamma} \eta_{\varepsilon}|D \varphi|^{2} \mathrm{~d} x .
$$

Since $\Re e\left(Y_{0}\right)>0$ we deduce that $\left(\varphi, w_{n}\right)_{\Sigma^{ \pm}}=0 \forall n \leq N$ (this proves that the solutions of the homogeneous problem (3.5) have an exponential decay at infinity) and that $D \varphi=0$ in $L^{2}(\Gamma)$. Therefore $S_{ \pm}^{p} \varphi=0$ and

$$
\frac{Y_{0}}{i k} \int_{\Gamma} \eta_{\varepsilon} D \varphi \overline{D \psi} \mathrm{d} x=0 .
$$

Remark 3.6. In the unphysical case $\Re e\left(Y_{0}\right) \leq 0$ Lemma 3.5 does not apply.

We can now conclude this paragraph with the

Theorem 3.7. Problem $\left(P_{\varepsilon}\right)$ is well-posed.

Proof. By Fredholm alternative, problem $\left(P_{\varepsilon}\right)$ is well-posed if and only if the homogeneous problem (3.6) has no solution except the trivial one $\varphi=0$. Such a solution can be extended to a solution $w$ in the unbounded domain $\Omega_{\infty}$, defined by

Find $w \in H^{1}\left(\Omega_{\infty}\right)$ such that $a^{\prime \prime}(w, \psi)=0 \quad \forall \psi \in H^{1}\left(\Omega_{\infty}\right)$,

where

$$
a^{\prime \prime}(w, \psi)=\int_{\Omega_{\infty}}(\nabla w \cdot \nabla \bar{\psi}-D w \overline{D \psi}) \mathrm{d} x \mathrm{~d} y .
$$

This extension $w$ of $\varphi$ is simply

$$
\begin{cases}w=\left.\varphi\right|_{\Omega} & \text { in } \Omega, \\ w=\sum_{n>N}\left(\varphi, w_{n}\right)_{\Sigma_{ \pm}} \mathrm{e}^{\mathrm{i} \beta_{n}^{ \pm}(x \mp R)} w_{n}(y) & \text { for } \pm x>R .\end{cases}
$$

Note that $w \in H^{1}\left(\Omega_{\infty}\right)$ since the serie expansions just involve evanescent modes. Therefore we have to find $w \in H^{1}\left(\Omega_{\infty}\right)$ such that

$$
\begin{aligned}
-\Delta w+D^{2} w & =0 \text { in } \mathcal{D}^{\prime}\left(\Omega_{\infty}\right), \\
\frac{\partial w}{\partial n} & =0 \quad \text { on } \partial \Omega_{\infty} .
\end{aligned}
$$


To conclude we just have to show that $w=0$. The horizontal Fourier transform $\hat{w}(\xi, y)$ satisfies for all $\xi \in \mathbb{R}$

$$
\begin{aligned}
& \left.-\frac{\mathrm{d}^{2} \hat{w}}{\mathrm{~d} y^{2}}=\left[(M \xi-k)^{2}-\xi^{2}\right] \hat{w} \text { for } \quad y \in\right] 0, h[, \\
& \frac{\mathrm{d} \hat{w}}{\mathrm{~d} y}=0 \quad \text { at } y=0 \text { and } h .
\end{aligned}
$$

Decomposing $\hat{w}$ on the transverse $L^{2}$-basis $\left(w_{n}\right)_{n \in \mathbb{N}}$, it is easy to prove that $\hat{w}=0$ for nearly every values of $\xi$ $\left(\hat{w} \neq 0\right.$ only if $\left.(M \xi-k)^{2}-\xi^{2}=(n \pi / h)^{2}, n \in \mathbb{N}\right)$.

\section{Convergence Results}

We will now consider the limit $\varepsilon \rightarrow 0$. First we will determine the limit problem and then we will prove the convergence of the solution of the problem $P_{\varepsilon}$ toward the solution of the limit problem $P_{0}$.

\subsection{The limit problem}

To prove the convergence of $\varphi_{\varepsilon}$ as $\varepsilon$ tends to 0 , we have to derive (formally) the "limit" problem (for $\varepsilon=0$ ) and to prove its well-posedness. Its solution will be proved in Paragraph 4.2 to be the limit of the sequence $\varphi_{\varepsilon}$. Defining the limit space

$$
V_{0}=\left\{\varphi \in H^{1}(\Omega), D\left(\left.\varphi\right|_{\Gamma}\right) \in L^{2}(\Gamma)\right\}
$$

the limit problem $\left(\mathrm{P}_{0}\right)$ is obviously:

Find $\varphi_{0} \in V_{0}$ such that

$$
a_{0}\left(\varphi_{0}, \psi\right)=\int_{\Omega} f \bar{\psi} \mathrm{d} x \mathrm{~d} y \text { for all } \psi \in V_{0}
$$

where for all $\varphi$ and $\psi \in V_{0}$

$$
a_{0}(\varphi, \psi)=\int_{\Omega}(\nabla \varphi \cdot \nabla \bar{\psi}-D \varphi \overline{D \psi}) \mathrm{d} x \mathrm{~d} y+\left\langle S_{ \pm} \varphi, \psi\right\rangle_{ \pm}+\frac{Y_{0}}{i k} \int_{\Gamma} D \varphi \overline{D \psi} \mathrm{d} x .
$$

Remark 4.1. The strong associated problem is found to be:

Find $\varphi \in V_{0}$ such that

$$
\begin{cases}-\Delta \varphi+D^{2} \varphi=f, & \text { in } \Omega, \\ \frac{\partial \varphi}{\partial n}=0, & \text { on } \Gamma_{0}, \\ \frac{\partial \varphi}{\partial n}=-T_{ \pm} \varphi, & \text { on } \Sigma^{ \pm}, \\ \frac{\partial \varphi}{\partial n}=\frac{Y_{0}}{i k} D^{2} \varphi, & \text { on } \Gamma, \\ D \varphi\left(0^{+}, h\right)=0=D \varphi\left(1^{-}, h\right) . & \end{cases}
$$

Only the boundary conditions at the liner ends $x=0$ and $x=1$ was not easy to guess. Note that such conditions can be seen as Kutta-like conditions.

As for the regularized problem we have the results

(1) The space $V_{0}$ defined in (4.1) equipped with the norm $\|\varphi\|_{0}^{2}=\|\varphi\|_{H^{1}(\Omega)}^{2}+\left\|D\left(\left.\varphi\right|_{\Gamma}\right)\right\|_{L^{2}(\Gamma)}^{2}$ is an Hilbert space.

(2) Problem $\left(P_{0}\right)$ is of Fredholm type and is well-posed.

Now we will prove that $\mathrm{P}_{\varepsilon} \rightarrow \mathrm{P}_{0}$ when $\varepsilon \rightarrow 0$. The main difficulty lies in the variational spaces: for all $\varepsilon>0$ the problem $\mathrm{P}_{\varepsilon}$ is defined in the space $V$ (defined in Eq. (3.4)) independent of $\varepsilon$ while $\mathrm{P}_{0}$ is defined in $V_{0}$ (defined in Eq. (4.1)) with $V_{0} \subset V, V_{0} \neq V$. Therefore there is no continuous transition for the solution spaces. 


\subsection{Convergence of the regularized problem}

First we will prove that the solution $\varphi_{\varepsilon}$ of $\mathrm{P}_{\varepsilon}$ converges weakly to the solution $\varphi_{0}$ of $\mathrm{P}_{0}$ when $\varepsilon \rightarrow 0$. To prove the strong convergence, the key point will be to prove that $V_{0}$ is dense in $V$ (Lem. 4.6).

Before taking the limit $\varepsilon \rightarrow 0$ let us introduce a definition: for all $\varphi \in V$

$$
\|\varphi\|_{\varepsilon}^{2}=\|\varphi\|_{H^{1}(\Omega)}^{2}+\left\|\eta_{\varepsilon}^{\frac{1}{2}} D\left(\left.\varphi\right|_{\Gamma}\right)\right\|_{L^{2}(\Gamma)}^{2} .
$$

Remark 4.2. Although the norm $\|\varphi\|_{\varepsilon}$ is not well adapted to the limit process $\varepsilon \rightarrow 0$ since it depends on $\varepsilon$, it will turn out to be useful since it appears naturally in the variational formulation. Note that for all $\varphi \in V$

$$
\|\varphi\|_{V} \leq\|\varphi\|_{\varepsilon}
$$

and moreover for all $\varphi \in V_{0} \subset V$

$$
\|\varphi\|_{V} \leq\|\varphi\|_{\varepsilon} \leq\|\varphi\|_{0}
$$

since $\eta \leq \eta_{\varepsilon} \leq 1$. Therefore for any sequence $\varphi_{\varepsilon} \in V$, if $\left\|\varphi_{\varepsilon}-\varphi\right\|_{\varepsilon} \rightarrow 0$ as $\varepsilon \rightarrow 0$, then $\varphi \in V$ but this does not imply that $\varphi \in V_{0}$. This is the complicated point of our study.

Now we will prove the

Theorem 4.3. If $\varphi_{\varepsilon} \in V$ is the solution of $\left(P_{\varepsilon}\right)$ and $\varphi_{0} \in V_{0}$ the solution of $\left(P_{0}\right)$, then $\left\|\varphi_{\varepsilon}-\varphi_{0}\right\|_{\varepsilon}^{2}=$ $\left\|\varphi_{\varepsilon}-\varphi_{0}\right\|_{H^{1}(\Omega)}^{2}+\left\|\eta_{\varepsilon}^{\frac{1}{2}} D\left(\varphi_{\varepsilon}-\varphi_{0}\right)\right\|_{L^{2}(\Gamma)}^{2} \rightarrow 0$ as $\varepsilon \rightarrow 0$.

In this aim we proceed in three steps: in the first step, we suppose that the sequence $\varphi_{\varepsilon} \in V$ is such that $\left\|\varphi_{\varepsilon}\right\|_{\varepsilon}$ is bounded and prove that it converges weakly to $\varphi_{0}$. In the second step, we prove that the convergence is strong. Finally in the third step, we prove that the hypothesis of the first step (boundedness of $\varphi_{\varepsilon}$ ) is necessarily true, which achieves the proof.

\subsubsection{Weak convergence}

Let us assume that the sequence $\varphi_{\varepsilon} \in V$ is such that $\left\|\varphi_{\varepsilon}\right\|_{\varepsilon}$ is bounded. Then due to the definition (4.2) of $\|\cdot\|_{\varepsilon}$, it is deduced that we can extract a sequence, denoted also $\varphi_{\varepsilon}$, which satisfies: there exists $\varphi \in H^{1}(\Omega)$ and $w \in L^{2}(\Gamma)$ such that

$$
\left\{\begin{array}{l}
\varphi_{\varepsilon} \rightarrow \varphi \text { in } H^{1}(\Omega), \\
\eta_{\varepsilon}^{\frac{1}{2}} D\left(\left.\varphi_{\varepsilon}\right|_{\Gamma}\right) \rightarrow w \text { in } L^{2}(\Gamma) .
\end{array}\right.
$$

We will show now that $w=D\left(\left.\varphi\right|_{\Gamma}\right)$ in $L^{2}(\Gamma)$. In this aim we will prove that $\eta_{\varepsilon}^{\frac{1}{2}} D\left(\left.\varphi_{\varepsilon}\right|_{\Gamma}\right) \rightarrow D\left(\left.\varphi\right|_{\Gamma}\right)$ in $\mathcal{D}^{\prime}(\Gamma)$. Thanks to the continuity of the trace application from $H^{1}(\Omega)$ into $H^{\frac{1}{2}}(\Gamma)$ and thanks to the compact embedding of $H^{\frac{1}{2}}(\Gamma)$ into $L^{2}(\Gamma)$ we deduce that

$$
\left.\left.\varphi_{\varepsilon}\right|_{\Gamma} \rightarrow \varphi\right|_{\Gamma} \text { in } L^{2}(\Gamma) .
$$

It follows that $\left.\left.\eta_{\varepsilon}^{\frac{1}{2}} \varphi_{\varepsilon}\right|_{\Gamma} \rightarrow \varphi\right|_{\Gamma}$ in $L^{2}(\Gamma)$ since

$$
\begin{aligned}
\left\|\eta_{\varepsilon}^{\frac{1}{2}} \varphi_{\varepsilon}-\varphi\right\|_{L^{2}(\Gamma)} & \leq\left\|\eta_{\varepsilon}^{\frac{1}{2}}\left(\varphi_{\varepsilon}-\varphi\right)\right\|_{L^{2}(\Gamma)}+\left\|\left(\eta_{\varepsilon}^{\frac{1}{2}}-1\right) \varphi\right\|_{L^{2}(\Gamma)}, \\
& \leq\left\|\varphi_{\varepsilon}-\varphi\right\|_{L^{2}(\Gamma)}+\left(\int_{\Gamma}\left(\eta_{\varepsilon}^{\frac{1}{2}}-1\right)^{2}|\varphi|^{2} \mathrm{~d} x\right)^{\frac{1}{2}},
\end{aligned}
$$

the last term tending to 0 thanks to Lebesgue's theorem. In addition since $\left.\left.\varphi_{\varepsilon}\right|_{\Gamma} \rightarrow \varphi\right|_{\Gamma}$ in $\mathcal{D}^{\prime}(\Gamma)$ we deduce that

$$
\frac{\partial}{\partial x}\left(\left.\varphi_{\varepsilon}\right|_{\Gamma}\right) \rightarrow \frac{\partial}{\partial x}\left(\left.\varphi\right|_{\Gamma}\right) \text { in } \mathcal{D}^{\prime}(\Gamma)
$$


and also that

$$
\eta_{\varepsilon}^{\frac{1}{2}} \frac{\partial}{\partial x}\left(\left.\varphi_{\varepsilon}\right|_{\Gamma}\right) \rightarrow \frac{\partial}{\partial x}\left(\left.\varphi\right|_{\Gamma}\right) \text { in } \mathcal{D}^{\prime}(\Gamma)
$$

Indeed $\forall \phi \in \mathcal{D}(\Gamma), \eta_{\varepsilon}^{\frac{1}{2}} \phi=\phi$ for $\varepsilon$ small enough (as soon as $\left.\operatorname{Supp}(\phi) \subset\right] \varepsilon, 1-\varepsilon[$ ). Therefore

$$
\left\langle\eta_{\varepsilon}^{\frac{1}{2}} \frac{\partial \varphi_{\varepsilon}}{\partial x}, \phi\right\rangle=\int_{\Gamma} \eta_{\varepsilon}^{\frac{1}{2}} \frac{\partial \varphi_{\varepsilon}}{\partial x} \phi \mathrm{d} x=\int_{\Gamma} \frac{\partial \varphi_{\varepsilon}}{\partial x} \phi \mathrm{d} x=\left\langle\frac{\partial \varphi_{\varepsilon}}{\partial x}, \phi\right\rangle
$$

for $\varepsilon$ small enough. Note that $\int_{\Gamma} \frac{\partial \varphi_{\varepsilon}}{\partial x} \phi \mathrm{d} x$ is defined since $\left.\varphi_{\varepsilon}\right|_{\Gamma} \in H_{l o c}^{1}(\Gamma)$. To conclude we just have to notice that

$$
\eta_{\varepsilon}^{\frac{1}{2}} D\left(\left.\varphi_{\varepsilon}\right|_{\Gamma}\right) \rightarrow w \text { in } \mathcal{D}^{\prime}(\Gamma)
$$

which implies that $D\left(\left.\varphi\right|_{\Gamma}\right)=w$ in $\mathcal{D}^{\prime}(\Gamma)$ and consequently in $L^{2}(\Gamma)$.

To prove that $\varphi=\varphi_{0}$ and that all the sequence $\varphi_{\varepsilon}$ converges, we will show that $\varphi$ solves $\mathrm{P}_{0}\left(P_{0}\right)$. Let $\psi$ be in $V$. We want to take the limit of problem $P_{\varepsilon}\left(P_{\varepsilon}\right)$ when $\varepsilon \rightarrow 0$. From $\eta_{\varepsilon} D\left(\left.\varphi_{\varepsilon}\right|_{\Gamma}\right) \rightarrow D\left(\left.\varphi\right|_{\Gamma}\right)$ in $\mathcal{D}^{\prime}(\Gamma)$, we deduce that $\eta_{\varepsilon} D\left(\left.\varphi_{\varepsilon}\right|_{\Gamma}\right) \rightarrow D\left(\left.\varphi\right|_{\Gamma}\right)$ in $L^{2}(\Gamma)$ thanks to the density of $\mathcal{D}(\Gamma)$ in $L^{2}(\Gamma)$. Therefore, using the fact that $\varphi_{\varepsilon} \rightarrow \varphi$ in $H^{1}(\Omega)$, by weak convergence we get for $\varepsilon \rightarrow 0$

$$
a_{\varepsilon}\left(\varphi_{\varepsilon}, \psi\right) \rightarrow a_{0}(\varphi, \psi) \quad \forall \psi \in V .
$$

Remark 4.4. We have just proved the weak convergence $\varphi_{\varepsilon} \rightarrow \varphi_{0}$ in the sense

$$
\left\{\begin{array}{l}
\varphi_{\varepsilon} \rightarrow \varphi_{0} \text { in } H^{1}(\Omega) \\
\eta_{\varepsilon}^{\frac{1}{2}} D\left(\left.\varphi_{\varepsilon}\right|_{\Gamma}\right) \rightarrow D\left(\left.\varphi_{0}\right|_{\Gamma}\right) \text { in } L^{2}(\Gamma)
\end{array}\right.
$$

We want to prove now that this convergence is a strong one. It is sufficient to prove that $\left\|\varphi_{\varepsilon}-\varphi_{0}\right\|_{\varepsilon} \rightarrow 0$ : indeed $\eta_{\varepsilon}^{\frac{1}{2}}\left[D\left(\left.\varphi\right|_{\Gamma}\right)-D\left(\left.\varphi_{0}\right|_{\Gamma}\right)\right] \rightarrow 0$ in $L^{2}(\Gamma)$ implies that $\eta_{\varepsilon}^{\frac{1}{2}} D\left(\left.\varphi\right|_{\Gamma}\right) \rightarrow D\left(\left.\varphi_{0}\right|_{\Gamma}\right)$ in $L^{2}(\Gamma)$ since

$$
\int_{\Gamma} \eta_{\varepsilon}\left|D \varphi_{0}\right|^{2} \rightarrow \int_{\Gamma}\left|D \varphi_{0}\right|^{2}
$$

thanks to Lebesgue's theorem. We have been unable to prove directly the strong convergence because $V_{0} \subset V$, $V_{0} \neq V$. Indeed starting from $a_{0}\left(\varphi_{0}, \psi\right)=\int_{\Omega} f \bar{\psi} \mathrm{d} x \mathrm{~d} y=a_{\varepsilon}\left(\varphi_{\varepsilon}, \psi\right)$ for all $\psi \in V_{0}$ and introducing the spliting $\varphi_{\varepsilon}=\varphi_{\varepsilon}-\varphi_{0}+\varphi_{0}$ it is easily deduced that

$$
\begin{aligned}
a_{\varepsilon}\left(\varphi_{\varepsilon}-\varphi_{0}, \psi\right) & =a_{0}\left(\varphi_{0}, \psi\right)-a_{\varepsilon}\left(\varphi_{0}, \psi\right), \\
& =\frac{Y_{0}}{i k} \int_{\Gamma}\left(D \varphi_{0}-\eta_{\varepsilon} D \varphi_{0}\right) \overline{D \psi} \mathrm{d} x .
\end{aligned}
$$

To prove that $\left\|\varphi_{\varepsilon}-\varphi_{0}\right\|_{\varepsilon} \rightarrow 0$ the usual last step is to take $\psi=\varphi_{\varepsilon}-\varphi_{0}$. However this is not possible in $a_{0}(\cdot, \cdot)$ because $\varphi_{\varepsilon}-\varphi_{0} \notin V_{0}$. In the following paragraph we explain how we proceeded to prove the strong convergence.

\subsubsection{Strong convergence}

Now the strong convergence of $\varphi_{\varepsilon}$ to $\varphi_{0}$ will be proved. This is achieved by introducing an auxiliary sequence between $\varphi_{\varepsilon}$ and $\varphi_{0}$ called $\tilde{\varphi}_{\varepsilon} \in V_{0}$ which will tend to both these quantities. To build this sequence, we have first proved that $V_{0}$ is dense in $V$. This is done by defining concretely for any element of $V$ a sequence of $V_{0}$ tending to this element. $\tilde{\varphi}_{\varepsilon}$ will be built in a similar way.

The difference between $V$ and $V_{0}$ is the regularity on $\Gamma$. For any $\varphi \in V,\left.\varphi\right|_{\Gamma}$ belongs to the space $W$ defined by

$$
W=\left\{v \in H^{\frac{1}{2}}(\Gamma), \eta^{\frac{1}{2}} \frac{\mathrm{d} v}{\mathrm{~d} x} \in L^{2}(\Gamma)\right\},
$$




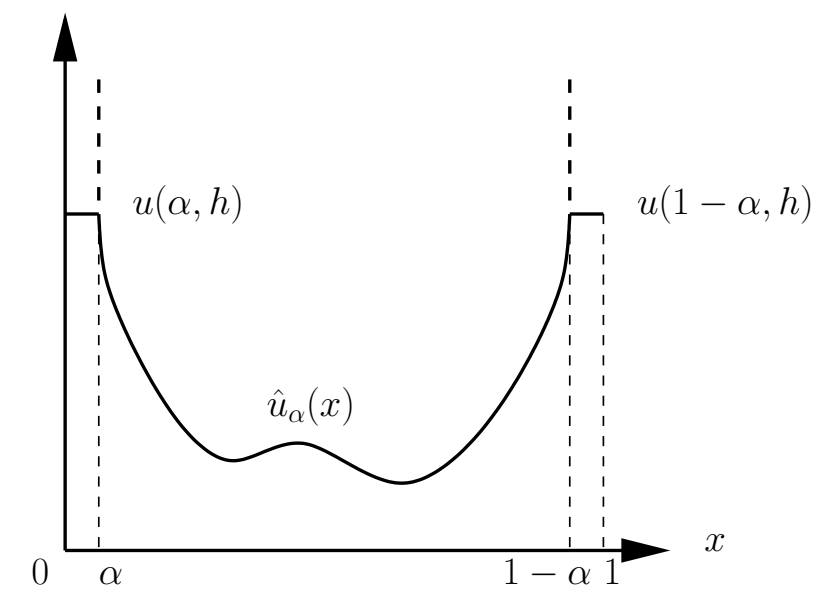

Figure 3. Construction of $\hat{\varphi}_{\alpha} \in H^{1}(\Gamma)$ from $\varphi \in W$.

equipped with the norm

$$
\|v\|_{W}^{2}=\|v\|_{H^{\frac{1}{2}}(\Gamma)}^{2}+\left\|\eta^{\frac{1}{2}} \frac{\mathrm{d} v}{\mathrm{~d} x}\right\|_{L^{2}(\Gamma)}^{2},
$$

whereas $\left.\varphi\right|_{\Gamma} \in H^{1}(\Gamma)$ when $\varphi \in V_{0}$. Therefore to prove that $V_{0}$ is dense in $V$ we will first focus on the trace behaviors and prove the

Theorem 4.5. The space $H^{1}(\Gamma)$ is dense in $W$ defined in (4.3).

Proof. Let us take $\varphi \in W$. For all $0<\alpha<1 / 2$ we define $\hat{\varphi}_{\alpha} \in H^{1}(\Gamma)$ such that

$$
\begin{cases}\hat{\varphi}_{\alpha}(x)=\varphi(x) & \text { on } \left.\Gamma_{\alpha}=\right] \alpha, 1-\alpha[, \\ \hat{\varphi}_{\alpha}(x)=\varphi(\alpha) & \text { on } \left.\Gamma_{\alpha}^{-}=\right] 0, \alpha[, \\ \hat{\varphi}_{\alpha}(x)=\varphi(1-\alpha) & \text { on } \left.\Gamma_{\alpha}^{+}=\right] 1-\alpha, 1[.\end{cases}
$$

$\varphi(\alpha)$ and $\varphi(1-\alpha)$ are defined since $\varphi \in H_{l o c}^{1}(\Gamma)$ (see Fig. 3). We introduce $\hat{v}_{\alpha}=\varphi-\hat{\varphi}_{\alpha} \in W$, explicitely defined by

$$
\left\{\begin{aligned}
\hat{v}_{\alpha}(x) & =0 & & \text { on } \Gamma_{\alpha}, \\
\hat{v}_{\alpha}(x) & =\varphi(x)-\varphi(\alpha) & & \text { on } \Gamma_{\alpha}^{-}, \\
\hat{v}_{\alpha} & =\varphi(x)-\varphi(1-\alpha) & & \text { on } \Gamma_{\alpha}^{+} .
\end{aligned}\right.
$$

We just need to prove that $\hat{v}_{\alpha} \rightarrow 0$ in $W$ when $\alpha \rightarrow 0$. In this aim we will need two results, proved in the appendix:

(1) Lemma A.1. there exists $C>0$ such that $\forall v \in W$ defined in (4.3),

$$
\|v\|_{H^{\frac{1}{2}}(\Gamma)}^{2} \leq C\left(\|v\|_{L^{2}(\Gamma)}^{2}+\left\|\eta^{\frac{1}{2}} \frac{\mathrm{d} v}{\mathrm{~d} x}\right\|_{L^{2}(\Gamma)}^{2}\right) .
$$


More precisely Lemma A.1 indicates that $W=\left\{v \in L^{2}(\Gamma), \eta^{\frac{1}{2}} \frac{\mathrm{d} v}{\mathrm{~d} x} \in L^{2}(\Gamma)\right\}$.

(2) Lemma A.2. For all $\varphi \in V, \lim _{x \rightarrow 0} x^{\frac{1}{2}} \varphi(x)=0=\lim _{x \rightarrow 1}(1-x)^{\frac{1}{2}} \varphi(x)$.

Therefore we have

$$
\left\|\hat{v}_{\alpha}\right\|_{H^{\frac{1}{2}(\Gamma)}}^{2} \leq C\left(\left\|\hat{v}_{\alpha}\right\|_{L^{2}(\Gamma)}^{2}+\left\|\eta^{\frac{1}{2}} \frac{\mathrm{d} \hat{v}_{\alpha}}{\mathrm{d} x}\right\|_{L^{2}(\Gamma)}^{2}\right)
$$

where

$$
\begin{aligned}
\left\|\hat{v}_{\alpha}\right\|_{L^{2}(\Gamma)}^{2}+\left\|\eta^{\frac{1}{2}} \frac{\mathrm{d} \hat{v}_{\alpha}}{\mathrm{d} x}\right\|_{L^{2}(\Gamma)}^{2} & =\int_{\Gamma_{\alpha}^{-}}|\varphi(x)-\varphi(\alpha)|^{2} \mathrm{~d} x+\int_{\Gamma_{\alpha}^{+}}|\varphi(x)-\varphi(1-\alpha)|^{2} \mathrm{~d} x+\int_{\Gamma_{\alpha}^{ \pm}} \eta\left|\frac{\partial \varphi}{\partial x}\right|^{2} \mathrm{~d} x \\
& \leq 2\left(\alpha|\varphi(\alpha)|^{2}+\alpha|\varphi(1-\alpha)|^{2}+\int_{\Gamma_{\alpha}^{ \pm}}|\varphi(x)|^{2} \mathrm{~d} x\right)+\int_{\Gamma_{\alpha}^{ \pm}} \eta\left|\frac{\partial \varphi}{\partial x}\right|^{2} \mathrm{~d} x
\end{aligned}
$$

The integrals tend to zero thanks to Lebesgue's theorem, the other terms tending to zero thanks to the Lemma A.2.

A direct consequence of Theorem 4.5 is the

Lemma 4.6. The space $V_{0}$ is dense in $V$.

Proof. Let us take $\varphi \in V$. Then $\left.\varphi\right|_{\Gamma} \in W$ defined in (4.3) and following the proof of Theorem 4.5, if for all $0<\alpha<1 / 2$ we define $\hat{\varphi}_{\alpha} \in H^{1}(\Gamma)$ such that

$$
\begin{cases}\hat{\varphi}_{\alpha}(x)=\varphi(x, h) & \text { for } \left.x \in \Gamma_{\alpha}=\right] \alpha, 1-\alpha[, \\ \hat{\varphi}_{\alpha}(x)=\varphi(\alpha, h) & \text { for } \left.x \in \Gamma_{\alpha}^{-}=\right] 0, \alpha[, \\ \hat{\varphi}_{\alpha}(x)=\varphi(1-\alpha, h) & \text { for } \left.x \in \Gamma_{\alpha}^{+}=\right] 1-\alpha, 1[,\end{cases}
$$

then $\hat{v}_{\alpha}=\left.\varphi\right|_{\Gamma}-\hat{\varphi}_{\alpha} \rightarrow 0$ in $W$. Now we introduce $\tilde{\varphi}_{\alpha}$ the unique solution in $V_{0}$ of

$$
\left\{\begin{aligned}
\left(\tilde{\varphi}_{\alpha}, \psi\right)_{H^{1}(\Omega)} & =(\varphi, \psi)_{H^{1}(\Omega)} \text { for all } \psi \in H^{1}(\Omega), \psi=0 \text { on } \Gamma, \\
\left.\tilde{\varphi}_{\alpha}\right|_{\Gamma} & =\hat{\varphi}_{\alpha} \text { on } \Gamma .
\end{aligned}\right.
$$

We just need to prove that $e_{\alpha}=\varphi-\tilde{\varphi}_{\alpha} \rightarrow 0$ in $V$ when $\alpha \rightarrow 0 . e_{\alpha}$ is the unique solution in $V$ of

$$
\left\{\begin{aligned}
\left(e_{\alpha}, \psi\right)_{H^{1}(\Omega)} & =0 \text { for all } \psi \in H^{1}(\Omega), \psi=0 \text { on } \Gamma, \\
\left.e_{\alpha}\right|_{\Gamma} & =\hat{v}_{\alpha} \text { on } \Gamma .
\end{aligned}\right.
$$

Since problem (4.4) is well-posed, there exists $C>0$ such that

$$
\left\|e_{\alpha}\right\|_{H^{1}(\Omega)}^{2} \leq C\left\|\hat{v}_{\alpha}\right\|_{H^{\frac{1}{2}}(\Gamma)}^{2}
$$

which simply tends to zero. Finally we use

$$
\left\|e_{\alpha}\right\|_{V}^{2}=\left\|e_{\alpha}\right\|_{H^{1}(\Omega)}^{2}+\left\|\eta^{\frac{1}{2}} D e_{\alpha}\right\|_{L^{2}(\Gamma)}^{2},
$$


to get $e_{\alpha} \rightarrow 0$ in $V$. Indeed the last term reads

$$
\left\|\eta^{\frac{1}{2}} D \hat{v}_{\alpha}\right\|_{L^{2}(\Gamma)} \leq M\left\|\eta^{\frac{1}{2}} \frac{\mathrm{d} \hat{v}_{\alpha}}{\mathrm{d} x}\right\|_{L^{2}(\Gamma)}+k\left\|\hat{v}_{\alpha}\right\|_{L^{2}(\Gamma)}
$$

which has been found to tend to zero in the proof of Theorem 4.5.

Now we introduce the auxiliary sequence $\tilde{\varphi}_{\varepsilon} \in V_{0}$ which will be used throughout all the rest of the section. For all $0<\varepsilon<1 / 2$ and all $\varphi_{\varepsilon} \in V$, proceeding similarly as in the proof of Lemma 4.6, we note $\tilde{\varphi}_{\varepsilon}$ the unique solution in $V_{0}$ of

$$
\left\{\begin{aligned}
\left(\tilde{\varphi}_{\varepsilon}, \psi\right)_{H^{1}(\Omega)} & =\left(\varphi_{\varepsilon}, \psi\right)_{H^{1}(\Omega)} \text { for all } \psi \in H^{1}(\Omega), \psi=0 \text { on } \Gamma, \\
\left.\tilde{\varphi}_{\varepsilon}\right|_{\Gamma} & =\hat{\varphi}_{\varepsilon} \text { on } \Gamma .
\end{aligned}\right.
$$

Here $\hat{\varphi}_{\varepsilon} \in H^{1}(\Gamma)$ is defined by

$$
\begin{cases}\hat{\varphi}_{\varepsilon}(x)=\varphi_{\varepsilon}(x, h) & \text { for } \left.x \in \Gamma_{\varepsilon}=\right] \varepsilon, 1-\varepsilon[, \\ \hat{\varphi}_{\varepsilon}(x)=\varphi_{\varepsilon}(\varepsilon, h) & \text { for } \left.x \in \Gamma_{\varepsilon}^{-}=\right] 0, \varepsilon[, \\ \hat{\varphi}_{\varepsilon}(x)=\varphi_{\varepsilon}(1-\varepsilon, h) & \text { for } \left.x \in \Gamma_{\varepsilon}^{+}=\right] 1-\varepsilon, 1[.\end{cases}
$$

Before proving that $\left\|\varphi_{\varepsilon}-\varphi_{0}\right\|_{\varepsilon} \rightarrow 0$, it is proved in Appendix 7 successively that the auxiliary sequence satisfies $\left\|\varphi_{\varepsilon}-\tilde{\varphi}_{\varepsilon}\right\|_{\varepsilon} \rightarrow 0$ and $\left\|\varphi_{0}-\tilde{\varphi}_{\varepsilon}\right\|_{0} \rightarrow 0$. These proofs are close to the one of Lemma 4.6, but more difficult because of the definition of $\hat{\varphi}_{\varepsilon}: \hat{\varphi}_{\varepsilon}(\varepsilon)$ depends in two ways of $\varepsilon$.

Now we are able to prove the strong convergence result for the model problem:

Theorem 4.7. If $\varphi_{\varepsilon} \in V$, the sequence solution of $\left(P_{\varepsilon}\right)$, is such that $\left\|\varphi_{\varepsilon}\right\|_{\varepsilon}$ is bounded (see definition (4.2)), then $\left\|\varphi_{\varepsilon}-\varphi_{0}\right\|_{\varepsilon} \rightarrow 0$ where $\varphi_{\varepsilon}$ is solution of $\left(P_{\varepsilon}\right)$ and $\varphi_{0}$ the solution of $\left(P_{0}\right)$.

Proof. We have proved that if the sequence $\varphi_{\varepsilon} \in V$ solution of $\left(P_{\varepsilon}\right)$ is such that $\left\|\varphi_{\varepsilon}\right\|_{\varepsilon}$ is bounded then $\left\|\tilde{e}_{\varepsilon}\right\|_{\varepsilon}=\left\|\varphi_{\varepsilon}-\tilde{\varphi}_{\varepsilon}\right\|_{\varepsilon} \rightarrow 0$ and $\left\|e_{\varepsilon}\right\|_{0}=\left\|\varphi_{0}-\tilde{\varphi}_{\varepsilon}\right\|_{0} \rightarrow 0$ where $\varphi_{0} \in V_{0}$ is the solution of $\left(P_{0}\right)$. Therefore $\left\|\varphi_{\varepsilon}-\varphi_{0}\right\|_{\varepsilon} \leq\left\|\varphi_{\varepsilon}-\tilde{\varphi}_{\varepsilon}\right\|_{\varepsilon}+\left\|\tilde{\varphi}_{\varepsilon}-\varphi_{0}\right\|_{0} \rightarrow 0$.

Now, we must prove that $\varphi_{\varepsilon}$ is necessarily bounded. We proceed by contradiction. So we assume that there is a subsequence (noted also $\varphi_{\varepsilon}$ ) such that: $\left\|\varphi_{\varepsilon}\right\|_{\varepsilon} \rightarrow \infty$ and we set: $U_{\varepsilon}=\frac{\varphi_{\varepsilon}}{\left\|\varphi_{\varepsilon}\right\|_{\varepsilon}}$. It is obvious that $\left\|U_{\varepsilon}\right\|_{\varepsilon}$ is bounded. Moreover, it solves $\left(P_{\varepsilon}\right)$ with $f$ replaced by $\frac{f}{\left\|\varphi_{\varepsilon}\right\|_{\varepsilon}} \rightarrow 0$. With the arguments used in the previous steps, we prove that there exists $U_{0} \in V_{0}$ such that $\left\|U_{\varepsilon}-U_{0}\right\|_{\varepsilon} \rightarrow 0$, where $U_{0}$ is the solution of the homogeneous problem (3.5). By hypothesis, this solution is trivial. Then, we obtain a contradiction since

$$
\left\|U_{\varepsilon}\right\|_{\varepsilon} \rightarrow 0 \text { and }\left\|U_{\varepsilon}\right\|_{\varepsilon}=1
$$

\section{Other FORMUlations}

In Sections 3 and 4 we focused on the velocity potential formulation. We have proved that the solution $\varphi_{\varepsilon}$ for a continuous boundary condition has a limit when $\varepsilon \rightarrow 0$ and that this limit $\varphi_{0}$ for a discontinuous transition is well defined since it satisfies a well-posed problem. Now we will consider the pressure and displacement formulations. We will show that although for a continuous boundary condition the pressure and the displacement potential satisfy well-posed problems, we are unable to prove they converge when $\varepsilon \rightarrow 0$, due to the fact that the expected limits don't satisfy well-posed limit problems.

Now we define the regularized problems satisfied by the pressure $p_{\varepsilon}$ and by the displacement potential $\zeta_{\varepsilon}$ and determine their limits when $\varepsilon \rightarrow 0$. 


\subsection{Link between the velocity, the pressure and the displacement formulations}

We recall that the radiation problem for the three natural unknowns reads:

Find $u \in H^{1}(\Omega)\left(u=p_{\varepsilon}, \varphi_{\varepsilon}\right.$ or $\left.\zeta_{\varepsilon}\right)$ such that

$$
\begin{cases}-\Delta u+D^{2} u=f, & \text { in } \Omega, \\ \frac{\partial u}{\partial n}=0, & \text { on } \Gamma_{0}, \\ \frac{\partial u}{\partial n}=-T_{ \pm} u, & \text { on } \Sigma^{ \pm}, \\ \text {and on } \Gamma: & \\ \text { If } u=p_{\varepsilon}: \frac{\partial p_{\varepsilon}}{\partial n}=\frac{Y_{0}}{i k} D^{2}\left(\eta_{\varepsilon} p_{\varepsilon}\right), & \\ \text { If } u=\varphi_{\varepsilon}: \frac{\partial \varphi_{\varepsilon}}{\partial n}=\frac{Y_{0}}{i k} D\left(\eta_{\varepsilon} D \varphi_{\varepsilon}\right), & \\ \text { If } u=\zeta_{\varepsilon}: \frac{\partial \zeta_{\varepsilon}}{\partial n}=\frac{Y_{0}}{i k} \eta_{\varepsilon} D^{2} \zeta_{\varepsilon} . & \end{cases}
$$

The important point is that if $\varphi_{\varepsilon}$ is solution of the velocity potential formulation of problem (5.1), then $p_{\varepsilon}=$ $-D \varphi_{\varepsilon}$ is solution of the pressure formulation. In particular applying the operator $D$ to $\frac{\partial \varphi_{\varepsilon}}{\partial n}=\frac{Y_{0}}{i k} D\left(\eta_{\varepsilon} D \varphi_{\varepsilon}\right)$ leads to $\frac{\partial p_{\varepsilon}}{\partial n}=\frac{Y_{0}}{i k} D^{2}\left(\eta_{\varepsilon} p_{\varepsilon}\right)$. In a same way if $\zeta_{\varepsilon}$ is solution of the displacement formulation then $\varphi_{\varepsilon}=D \zeta_{\varepsilon}$ is solution of the velocity formulation.

The variational formulation of the radiation problem reads $a_{\varepsilon}(u, v)=\int_{\Omega} f \bar{v}$ where the sesquilinear form $a_{\varepsilon}(u, v)$ is defined by

- For the velocity potential

$$
a_{\varepsilon}(\varphi, \psi)=\int_{\Omega}(\nabla \varphi \cdot \nabla \bar{\psi}-D \varphi \overline{D \psi}) \mathrm{d} x \mathrm{~d} y+\left\langle S_{ \pm} \varphi, \psi\right\rangle_{ \pm}+\frac{Y_{0}}{i k} \int_{\Gamma} \eta_{\varepsilon} D \varphi \overline{D \psi} \mathrm{d} x
$$

- For the pressure

$$
a_{\varepsilon}(p, q)=\int_{\Omega}(\nabla p \cdot \nabla \bar{q}-D p \overline{D q}) \mathrm{d} x \mathrm{~d} y+\left\langle S_{ \pm} p, q\right\rangle_{ \pm}+\frac{Y_{0}}{i k} \int_{\Gamma}\left(\eta_{\varepsilon} D p \overline{D q}+M \frac{\mathrm{d} \eta_{\varepsilon}}{\mathrm{d} x} p \overline{D q}\right) \mathrm{d} x .
$$

- For the displacement potential

$$
a_{\varepsilon}(\zeta, \theta)=\int_{\Omega}(\nabla \zeta \cdot \nabla \bar{\theta}-D \zeta \overline{D \theta}) \mathrm{d} x \mathrm{~d} y+\left\langle S_{ \pm} \zeta, \theta\right\rangle_{ \pm}+\frac{Y_{0}}{i k} \int_{\Gamma}\left(\eta_{\varepsilon} D \zeta \overline{D \theta}+M \frac{\mathrm{d} \eta_{\varepsilon}}{\mathrm{d} x} D \zeta \bar{\theta}\right) \mathrm{d} x .
$$

Remark 5.1. To define well-posed variational formulations for $p_{\varepsilon}$ and $\zeta_{\varepsilon}$ we need more regularity for $\eta_{\varepsilon}$ and we choose $\eta_{\varepsilon} \in C^{1}(\Gamma)$. Also to get rid of the boundary terms on $\Gamma$ at $x=0$ and $x=1$ we need that $\eta_{\varepsilon}$ and $\mathrm{d} \eta_{\varepsilon} / \mathrm{d} x$ vanish at these two points. For the velocity potential model we just needed $\eta_{\varepsilon}$ to vanish.

Since the additional terms, compared to the velocity formulation, namely the integrals on $\Gamma$ of $M \eta_{\varepsilon}^{\prime} p \overline{D q}$ and of $M \eta_{\varepsilon}^{\prime} D \zeta \bar{\theta}$, are compact perturbations, it is straightforward to prove that the variational formulations for the pressure and the displacement potential are well-posed for any $\varepsilon>0$. However we are unable to deduce limit problems when $\varepsilon \rightarrow 0$. Indeed $\left|\mathrm{d} \eta_{\varepsilon} / \mathrm{d} x\right| \rightarrow \infty$ at $x=0$ and 1 when $\varepsilon \rightarrow 0$. But we can determine formally the limits of the pressure and of the displacement, which is done in the next paragraph. 


\subsection{Limit process for the pressure and the displacement solutions}

Using the links between the pressure, the displacement potential and the velocity potential we can determine the limits of $p_{\varepsilon}$ and $\zeta_{\varepsilon}$. Indeed if $\varphi_{\varepsilon}$ is solution of the velocity problem then $p_{\varepsilon}=-D \varphi_{\varepsilon}$ and $\zeta_{\varepsilon}$ is defined such that $\varphi_{\varepsilon}=D \zeta_{\varepsilon}$. Since $\varphi_{\varepsilon} \in V$ tends to $\varphi_{0} \in V_{0}$ in the sense

$$
\left\{\begin{array}{l}
\varphi_{\varepsilon} \rightarrow \varphi_{0} \text { in } H^{1}(\Omega), \\
\eta_{\varepsilon}^{\frac{1}{2}} D\left(\left.\varphi_{\varepsilon}\right|_{\Gamma}\right) \rightarrow D\left(\left.\varphi_{0}\right|_{\Gamma}\right) \text { in } L^{2}(\Gamma), \\
\text { with } D \varphi_{0}\left(0^{+}, h\right)=0=D \varphi_{0}\left(1^{-}, h\right),
\end{array}\right.
$$

we deduce immediatly some convergence results for $p_{\varepsilon}$ and $\zeta_{\varepsilon}$ :

- For the pressure

$$
\left\{\begin{array}{l}
p_{\varepsilon} \rightarrow p_{0} \text { in } L^{2}(\Omega) \\
\left.\left.\eta_{\varepsilon}^{\frac{1}{2}} p_{\varepsilon}\right|_{\Gamma} \rightarrow p_{0}\right|_{\Gamma} \text { in } L^{2}(\Gamma) \\
\text { with } p_{0}=-D \varphi_{0} \text { and } p_{0}\left(0^{+}, h\right)=0=p_{0}\left(1^{-}, h\right)
\end{array}\right.
$$

- For the displacement potential

$$
\left\{\begin{array}{l}
D \zeta_{\varepsilon} \rightarrow D \zeta_{0} \text { in } H^{1}(\Omega), \\
\eta_{\varepsilon}^{\frac{1}{2}} D\left[\left.\left(D \zeta_{\varepsilon}\right)\right|_{\Gamma}\right] \rightarrow D\left[\left.\left(D \zeta_{0}\right)\right|_{\Gamma}\right] \text { in } L^{2}(\Gamma), \\
\text { with } \varphi_{0}=D \zeta_{0} \text { and } D^{2} \zeta_{0}\left(0^{+}, h\right)=0=D^{2} \zeta_{0}\left(1^{-}, h\right)
\end{array}\right.
$$

Remembering that the natural space to define the variational formulations for the three unknowns is $H^{1}(\Omega)$, we understand now clearly why we cannot define variational problems satisfied by $p_{0}$ or $\zeta_{0}$ thanks to the limit process $\varepsilon \rightarrow 0$. $p_{0}$ is too weak, it belongs only to $L^{2}(\Omega)$. On the other hand $\zeta_{0}$ is too regular to be associated to a $H^{1}$ variational formulation.

Note that the Wiener-Hopf approach indicates that the pressure varies close to $x=0$ like $\sqrt{x}$ without the Kutta condition or $x^{3 / 2}$ with the Kutta condition [11,12], in accordance with our result $p_{0}\left(0^{+}, h\right)=0=$ $p_{0}\left(1^{-}, h\right)$.

\section{Numerical Results: Limit PROCESS $\varepsilon \rightarrow 0$}

Now we investigate numerically, thanks to the Finite Element code MELINA [30], the behavior of the three unknowns on the upper wall with the liner at $y=h$ when $\varepsilon \rightarrow 0$. We have used standard P2 Lagrange finite elements, which lead to a conforming approximation of $V$. On Figure 4 is plotted in solid line the real part of $\varphi_{\varepsilon}$ for decreasing values of $\varepsilon$. The liner is located at $] 0,3\left[. \varphi_{\varepsilon}\right.$ is found to tend to a continuous function. In dotted line is represented the real part of the convective derivative of $\varphi_{\varepsilon}$ : we confirm numerically that the velocity potential tends to a function $\varphi_{0}$ satisfying the local behavior $D \varphi_{0}\left(0^{+}, h\right)=0=D \varphi_{0}\left(3^{-}, h\right)$. Note that the behavior of $D \varphi_{0}$ seems to be singular outside the liner: $\left|D \varphi_{0}\left(0^{-}, h\right)\right|=\infty=\left|D \varphi_{0}\left(3^{+}, h\right)\right|$.

Figure 5 shows the pressure and the displacement potential for a small value $\varepsilon=0.01$ (we recall that we do not know how to define the limit problems for $\varepsilon=0$ for these unknowns). Contrary to Figure 4 where $\eta_{\varepsilon}$ defined in equation (3.2) was a $C^{0}(\mathbb{R})$ function, now $\eta_{\varepsilon}$ is chosen as a $C^{1}(\mathbb{R})$ function (see Rem. 5.1): it is an order 2 polynomial on $[0, \varepsilon]$ and on $[3-\varepsilon, 3]$. This means that $D \varphi_{\varepsilon}$ determined in Figure 4 should be different from $p_{\varepsilon}$ plotted on Figure $5 \mathrm{a}$, the relation $p_{\varepsilon}=D \varphi_{\varepsilon}$ being rigorously valid only if both quantities are defined with the same $\eta_{\varepsilon}$. In practice this is not the case and $p_{\varepsilon}$ plotted of Figure $5 \mathrm{a}$ is very close to $D \varphi_{\varepsilon}$ of Figure $4 \mathrm{c}$. This shows that the choice of the regularity of $\eta_{\varepsilon}$ does not seem to be sensitive in the limit process $\varepsilon \rightarrow 0$. Numerically the pressure is found to become singular outside the lined area when approaching a discontinuous transition. 
(a)

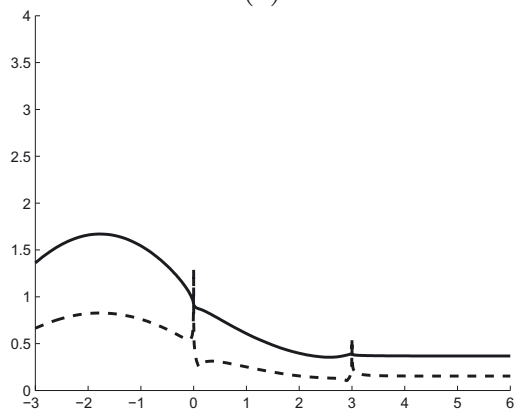

(b)

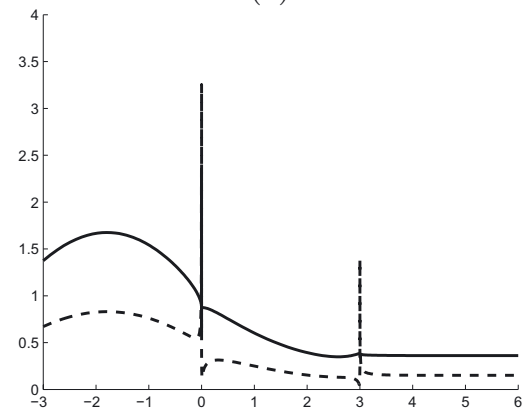

(c)

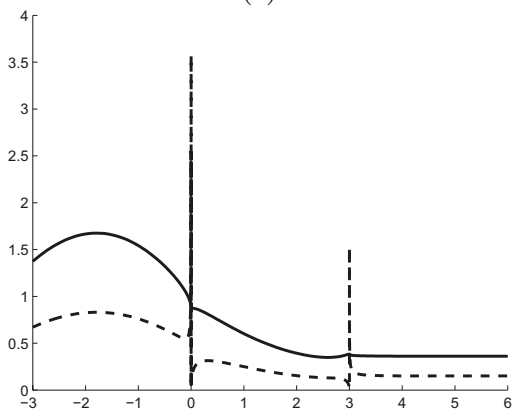

FiguRE 4. Solid line: $\Re e\left[\varphi_{\epsilon}(x, h)\right]$, dotted line: $\Re e\left[D \varphi_{\epsilon}(x, h)\right]$ for $(\mathrm{a}): \varepsilon=0.1$, (b): $\varepsilon=0.01$ and (c): $\varepsilon=0$.
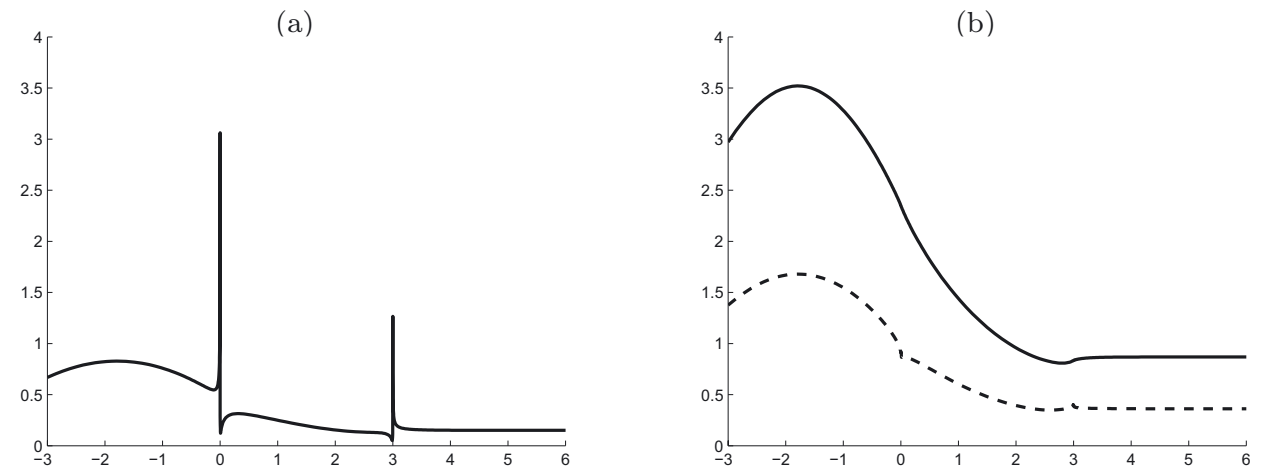

Figure 5. For $\varepsilon=0.01$, (a): $\Re e\left[p_{\epsilon}(x, h)\right]$, (b): solid line: $\Re e\left[\zeta_{\epsilon}(x, h)\right]$, dotted line: $\Re e\left[D \zeta_{\epsilon}(x, h)\right]$

Since the quality of the convergence of the finite element approximation (when the mesh size decreases) depends on the regularity of the solution, the precision of the approximation becomes bad. This confirms that the velocity formulation should be preferred to the pressure one. As expected the pressure tends to a function $p_{0}$ satisfying $p_{0}\left(0^{+}, h\right)=0=p_{0}\left(3^{-}, h\right)$.

Finally the displacement (Fig. 5b in solid line) tends to a regular function (in particular in $C^{1}(\mathbb{R})$ ). As expected $D \zeta_{\varepsilon}$ plotted in dotted line is very close to $\varphi_{\varepsilon}$. Note that both the velocity and the displacement potentials tend to continuous functions on the upper wall: we recover the edge conditions of the mode-matching methods [6], which impose some regularity to the velocity or to the displacement, not to the pressure. 


\section{Conclusion}

We have shown that to study the acoustic scattering in presence of a flow and of a discontinuous boundary condition between a hard wall and a lined wall, the velocity potential seems to be a better unknown to choose than the pressure or the displacement. Indeed although the three unknowns satisfy well-posed problems for a continuous boundary condition, only the velocity converges to the solution of a well-posed problem when the function discribing the boundary condition tends to a discontinuous function. Moreover some edge conditions must be considered for the velocity potential: its convective derivative must vanish at the liner ends.

An improvement of our study would be to take into account the presence of a vorticity line developing from the boundary discontinuities. This has been done in the case of the acoustic diffraction by a rigid plate in a duct in presence of a flow [31]: the usual modeling of viscous effects is that the pressure can be infinite at the plate trailing edge but it must be finite at the leading edge. To impose such regularity, a wake is introduced behind the plate and a Kutta condition is applied at the trailing edge to adjust the wake amplitude. This procedure is not straightforward to extend to the case of an impedance boundary condition instead of a rigid plate: we do not know any analytical expression of the wake, we do not know if the wake extends from the leading edge or only from the liner trailing edge and finally the expected behavior of the pressure at the liner ends is unknown.

Another way to study the scattering problem is to consider a shear flow with a varying Mach number profile $M(y)$ vanishing on the liner. Then the Myers boundary condition becomes simply $\partial u / \partial y=i k Y u$ for $u=p, \varphi$ or $\zeta$ and this leads to well posed problems even when $Y$ is discontinuous. However then the convected Helmholtz equation (2.3) is no longer valid. The full Euler equations must be considered, which complicates a lot the study.

\section{Appendix A. Regularity on the treated Boundary}

Here are demonstrated two lemmas necessary to prove the density of $V_{0}$ (defined in Eq. (4.1)) in $V$ (defined in Eq. (3.4)).

Lemma A.1. If $u \in W_{0}=\left\{u \in L^{2}(\Gamma) ; \eta^{\frac{1}{2}} \frac{\mathrm{d} u}{\mathrm{~d} x} \in L^{2}(\Gamma)\right\}$, then $u \in H^{\frac{1}{2}}(\Gamma)$ and there exists $C>0$ such that $\forall u \in W_{0}$

$$
\|u\|_{H^{\frac{1}{2}(\Gamma)}}^{2} \leq C\left(\|u\|_{L^{2}(\Gamma)}^{2}+\left\|\eta^{\frac{1}{2}} \frac{\mathrm{d} u}{\mathrm{~d} x}\right\|_{L^{2}(\Gamma)}^{2}\right) .
$$

Proof. Let us remark first that $W_{0} \subset H_{l o c}^{1}(\Gamma)$. Therefore we just need to prove that $x(\mathrm{~d} u / \mathrm{d} x)^{2}$ integrable in $x=0$ implies that $u$ is locally in $H^{\frac{1}{2}}$ close to 0 (the same for $(1-x)(\mathrm{d} u / \mathrm{d} x)^{2}$ close to 1$)$. In this aim we note $\left.\Gamma^{-}=\right] 0, \frac{1}{2}[$ (or $] 0, \delta[$ for any $0<\delta<1 / 2$ ) and we introduce the quarter of disk of radius $1 / 2$

$$
\Omega^{-}=\left\{(x, y) \in \mathbb{R}^{2} ; x^{2}+y^{2} \leq \frac{1}{4}, 0 \leq x \leq \frac{1}{2} \text { and } 0 \leq y \leq \frac{1}{2}\right\} .
$$

For all $u \in W_{0}$ (which implies $x^{\frac{1}{2}} \frac{\mathrm{d} u}{\mathrm{~d} x} \in L^{2}\left(\Gamma^{-}\right)$), we define in $\Omega^{-}$the function $v(x, y)=u\left(\sqrt{x^{2}+y^{2}}\right)$. It reads in polar coordinates $\tilde{v}(r, \theta)=v(r \cos \theta, r \sin \theta)=u(r) . v$ belongs to $H^{1}\left(\Omega^{-}\right)$since

$$
\|v\|_{H^{1}\left(\Omega^{-}\right)}^{2}=\frac{\pi}{4} \int_{0}^{\frac{1}{2}}\left(|u(r)|^{2}+\left|\frac{\mathrm{d} u}{\mathrm{~d} r}\right|^{2}\right) r \mathrm{~d} r \leq \frac{\pi}{4}\left(\int_{0}^{\frac{1}{2}} \frac{1}{2}|u(r)|^{2} \mathrm{~d} r+\int_{0}^{\frac{1}{2}}\left|\frac{\mathrm{d} u}{\mathrm{~d} r}\right|^{2} r \mathrm{~d} r\right),
$$

since $r \leq 1 / 2$. Thanks to the Trace theorem from $H^{1}\left(\Omega^{-}\right)$to $H^{\frac{1}{2}}\left(\Gamma^{-}\right)$we deduce that there exists $C>0$ such that

$$
\|u\|_{H^{\frac{1}{2}\left(\Gamma^{-}\right)}}^{2}=\left\|v_{\mid \Gamma^{-}}\right\|_{H^{\frac{1}{2}\left(\Gamma^{-}\right)}}^{2} \leq C^{\prime}\|v\|_{H^{1}\left(\Omega^{-}\right)}^{2} \leq C\left(\|u\|_{L^{2}\left(\Gamma^{-}\right)}^{2}+\left\|\eta^{\frac{1}{2}} \frac{\mathrm{d} u}{\mathrm{~d} x}\right\|_{L^{2}\left(\Gamma^{-}\right)}^{2}\right) .
$$


In a same way noting $\left.\Gamma^{+}=\right] \frac{1}{2}, 1[$ and introducing the change of variable $\tilde{x}=1-x$ we can prove that there exists $C>0$ such that

$$
\|u\|_{H^{\frac{1}{2}\left(\Gamma^{+}\right)}}^{2} \leq C\left(\|u\|_{L^{2}\left(\Gamma^{+}\right)}^{2}+\left\|\eta^{\frac{1}{2}} \frac{\mathrm{d} u}{\mathrm{~d} x}\right\|_{L^{2}\left(\Gamma^{+}\right)}^{2}\right) .
$$

Since $u \in H^{\frac{1}{2}}\left(\Gamma^{+}\right) \cap H^{\frac{1}{2}}\left(\Gamma^{-}\right) \cap H_{l o c}^{1}(\Gamma)$, it is easy to conclude using the definition

$$
\|u\|_{H^{\frac{1}{2}(\Gamma)}}^{2}=\int_{\Gamma} \int_{\Gamma}\left|\frac{u(x)-u(y)}{x-y}\right|^{2} \mathrm{~d} x \mathrm{~d} y+\|u\|_{L^{2}(\Gamma)}^{2} .
$$

Lemma A.2. For all $u \in W$ defined in (4.3), $\lim _{x \rightarrow 0} x^{\frac{1}{2}} u(x)=0=\lim _{x \rightarrow 1}(1-x)^{\frac{1}{2}} u(x)$.

Proof. We will just prove the result at $x=0$, the result at $x=1$ can be proved in a similar way. Let us take $u \in \mathcal{C}^{\infty}(\bar{\Gamma})$. For all $x \in[0,1]$,

$$
x^{\frac{1}{2}} u(x)=\int_{0}^{x} \frac{\mathrm{d}}{\mathrm{d} t}\left(t^{\frac{1}{2}} u(t)\right) \mathrm{d} t=\int_{0}^{x}\left(\frac{\mathrm{d} u}{\mathrm{~d} t} t^{\frac{1}{2}}+\frac{t^{-\frac{1}{2}}}{2} u(t)\right) \mathrm{d} t .
$$

Now we use Cauchy-Schwarz inequality to find an upper bound. Since $W \neq H_{00}^{\frac{1}{2}}(\Gamma)$, for $u \in W$ we do not have necessarily $t^{-\frac{1}{2}} u \in L^{2}(\Gamma)$. However $W \subset H^{\frac{1}{2}}(\Gamma)$ and we use Hardy's inequality for all $0<s<1 / 2$, there exists $C_{s}>0$ and $C_{s}^{\prime}>0$ such that for all $u \in H^{\frac{1}{2}}(\Gamma) \subset H^{s}(\Gamma)$

$$
\left\|\frac{u}{\eta^{s}}\right\|_{L^{2}(\Gamma)} \leq C_{s}\|u\|_{H^{s}(\Gamma)} \leq C_{s}^{\prime}\|u\|_{H^{\frac{1}{2}}(\Gamma)}
$$

This means that for all $0<s<1 / 2,\left(u / t^{s}\right)^{2}$ is integrable at $t=0$. Therefore for $x \leq 1 / 2$

$$
x^{\frac{1}{2}}|u(x)| \leq x^{\frac{1}{2}}\left(\int_{0}^{x} t\left|\frac{\mathrm{d} u}{\mathrm{~d} t}\right|^{2} \mathrm{~d} t\right)^{\frac{1}{2}}+\frac{1}{2} \frac{x^{s}}{\sqrt{2 s}}\left(\int_{0}^{x}\left|\frac{u}{t^{s}}\right|^{2} \mathrm{~d} t\right)^{\frac{1}{2}} \leq x^{\frac{1}{2}}\left\|\eta \frac{\mathrm{d} u}{\mathrm{~d} t}\right\|_{L^{2}(\Gamma)}+\frac{1}{2} \frac{x^{s}}{\sqrt{2 s}} C_{s}^{\prime}\|u\|_{H^{\frac{1}{2}}(\Gamma)} .
$$

Thus we get $\lim _{x \rightarrow 0} x^{\frac{1}{2}} u(x)=0$. This result remains valid in $W$ by density of $\mathcal{C}^{\infty}(\bar{\Gamma})$ in $W$.

\section{Appendix B. Limits of the auxiliary sequence}

Here are presented the technical proofs of the two lemmas characterizing the convergence of the auxiliary sequence $\tilde{\varphi}_{\varepsilon}$.

Lemma B.3. If $\varphi_{\varepsilon} \in V$, the sequence solution of $\left(P_{\varepsilon}\right)$, is such that $\left\|\varphi_{\varepsilon}\right\|_{\varepsilon}$ is bounded (see definition (4.2)), then $\left\|\varphi_{\varepsilon}-\tilde{\varphi}_{\varepsilon}\right\|_{\varepsilon} \rightarrow 0$ where $\tilde{\varphi}_{\varepsilon}$ is defined in (4.5).

Proof. We introduce $\tilde{e}_{\varepsilon}=\varphi_{\varepsilon}-\tilde{\varphi}_{\varepsilon}$ which is the unique solution in $V$ of

$$
\left\{\begin{aligned}
\left(\tilde{e}_{\varepsilon}, \psi\right)_{H^{1}(\Omega)} & =0 \text { for all } \psi \in H^{1}(\Omega), \psi=0 \text { on } \Gamma, \\
\left.\tilde{e}_{\varepsilon}\right|_{\Gamma} & =\hat{v}_{\varepsilon} \text { on } \Gamma
\end{aligned}\right.
$$

where $\hat{v}_{\varepsilon} \in W$ defined in (4.3) is such that

$$
\begin{cases}\hat{v}_{\varepsilon}(x)=0 & \text { for } \left.x \in \Gamma_{\varepsilon}=\right] \varepsilon, 1-\varepsilon[, \\ \hat{v}_{\varepsilon}(x)=\varphi_{\varepsilon}(x, h)-\varphi_{\varepsilon}\left(\varepsilon^{ \pm}, h\right) & \text { for } x \in \Gamma_{\varepsilon}^{ \pm}\end{cases}
$$


where $\varepsilon^{+}=\varepsilon$ and $\varepsilon^{-}=1-\varepsilon$ and where we note $\left.\varphi_{\varepsilon}\right|_{\Gamma}(x, h)$ instead of $\left.\varphi_{\varepsilon}\right|_{\Gamma}(x, h)$. We will show that the quantity

$$
\left\|\tilde{e}_{\varepsilon}\right\|_{\varepsilon}^{2}=\left\|\tilde{e}_{\varepsilon}\right\|_{H^{1}(\Omega)}^{2}+\left\|\eta_{\varepsilon}^{\frac{1}{2}} D \tilde{e}_{\varepsilon}\right\|_{L^{2}(\Gamma)}^{2}
$$

tends to zero. In this aim we will prove successively that

- $\tilde{e}_{\varepsilon} \rightarrow 0$ in $H^{1}(\Omega)$;

- $\int_{\Gamma_{\varepsilon}^{ \pm}} \eta_{\varepsilon}\left|\frac{\partial \varphi_{\varepsilon}}{\partial x}\right|^{2} \mathrm{~d} x \rightarrow 0$

- $\left\|\eta_{\varepsilon}^{\frac{1}{2}} D \tilde{e}_{\varepsilon}\right\|_{L^{2}(\Gamma)}^{2} \rightarrow 0$.

(1) Proof of $\tilde{e}_{\varepsilon} \rightarrow 0$ in $H^{1}(\Omega)$

To prove that $\tilde{e}_{\varepsilon}=\varphi_{\varepsilon}-\tilde{\varphi}_{\varepsilon} \rightarrow 0$ in $V$ we will need two lemmas, proved in the Appendix 7. Since problem (B.1) is well-posed and thanks to Lemma A.1 we have

$$
\left\|\tilde{e}_{\varepsilon}\right\|_{H^{1}(\Omega)}^{2} \leq C^{\prime}\left\|\hat{v}_{\varepsilon}\right\|_{H^{\frac{1}{2}(\Gamma)}}^{2} \leq C C^{\prime}\left(\left\|\hat{v}_{\varepsilon}\right\|_{L^{2}(\Gamma)}^{2}+\left\|\eta^{\frac{1}{2}} \frac{\mathrm{d} \hat{v}_{\varepsilon}}{\mathrm{d} x}\right\|_{L^{2}(\Gamma)}^{2}\right),
$$

with

$$
\begin{aligned}
\left\|\hat{v}_{\varepsilon}\right\|_{L^{2}(\Gamma)}^{2}+\left\|\eta^{\frac{1}{2}} \frac{\mathrm{d} \hat{v}_{\varepsilon}}{\mathrm{d} x}\right\|_{L^{2}(\Gamma)}^{2}= & \int_{\Gamma_{\varepsilon}^{-}}\left|\varphi_{\varepsilon}(x, h)-\varphi_{\varepsilon}(\varepsilon, h)\right|^{2} \mathrm{~d} x \\
& +\int_{\Gamma_{\varepsilon}^{+}}\left|\varphi_{\varepsilon}(x, h)-\varphi_{\varepsilon}(1-\varepsilon, h)\right|^{2} \mathrm{~d} x+\int_{\Gamma_{\varepsilon}^{ \pm}} \eta\left|\frac{\partial \varphi_{\varepsilon}}{\partial x}\right|^{2} \mathrm{~d} x, \\
\leq & 2\left(\varepsilon\left|\varphi_{\varepsilon}(\varepsilon, h)\right|^{2}+\varepsilon\left|\varphi_{\varepsilon}(1-\varepsilon, h)\right|^{2}+\int_{\Gamma_{\varepsilon}^{ \pm}}\left|\varphi_{\varepsilon}(x, h)\right|^{2} \mathrm{~d} x\right)+\int_{\Gamma_{\varepsilon}^{ \pm}} \eta\left|\frac{\partial \varphi_{\varepsilon}}{\partial x}\right|^{2} \mathrm{~d} x .
\end{aligned}
$$

The two non-integral terms are proved to tend to zero by adapting the proof a Lemma A.2 to the function $\varphi_{\varepsilon}$ depending on the parameter $\varepsilon$ tending to zero. Indeed thanks to Lemma A.2: $\forall \varepsilon>0$ and for all $0<s<1 / 2$ we have

$$
\varepsilon^{\frac{1}{2}}\left|\varphi_{\varepsilon}(\varepsilon, h)\right| \leq \varepsilon^{\frac{1}{2}}\left(\int_{0}^{\varepsilon} t\left|\frac{\partial \varphi_{\varepsilon}}{\partial t}\right|^{2} \mathrm{~d} t\right)^{\frac{1}{2}}+\frac{1}{2} \frac{\varepsilon^{s}}{\sqrt{2 s}}\left(\int_{0}^{\varepsilon}\left|\frac{\varphi_{\varepsilon}}{t^{s}}\right|^{2} \mathrm{~d} t\right)^{\frac{1}{2}} .
$$

Since $\left\|\varphi_{\varepsilon}\right\|_{\varepsilon}=\left\|\varphi_{\varepsilon}\right\|_{H^{1}(\Omega)}^{2}+\left\|\eta_{\varepsilon}^{\frac{1}{2}} D \varphi_{\varepsilon}\right\|_{L^{2}(\Gamma)}^{2}$ is bounded and using Hardy' inequality:

For all $0<s<1 / 2$, there exists $C_{s}^{\prime}>0$ such that for all $\varepsilon>0$

$$
\left\|\frac{\varphi_{\varepsilon}}{\eta^{s}}\right\|_{L^{2}(\Gamma)} \leq C_{s}^{\prime}\left\|\varphi_{\varepsilon}\right\|_{H^{\frac{1}{2}}(\Gamma)}
$$

is deduced that both terms

$$
\int_{0}^{\varepsilon} t\left|\frac{\partial \varphi_{\varepsilon}}{\partial t}\right|^{2} \mathrm{~d} t \quad \text { and } \quad \int_{0}^{\varepsilon}\left|\frac{\varphi_{\varepsilon}}{t^{s}}\right|^{2} \mathrm{~d} t
$$

are bounded since $t \leq t / \varepsilon=\eta_{\varepsilon}$ on $[0, \varepsilon]$.

Also since $\varphi_{\varepsilon} \rightarrow \varphi_{0}$ in $L^{2}(\Gamma)$, the first integral tends to zero thanks to Lebesgue's theorem. But contrary to what happened when proving the density result of Theorem 4.5, the second integral does not tend simply 
to zero thanks to Lebesgue's theorem. Indeed we just have the weak convergence $\eta_{\varepsilon}^{\frac{1}{2}} \frac{\partial}{\partial x}\left(\left.\varphi_{\varepsilon}\right|_{\Gamma}\right) \rightarrow \frac{\partial}{\partial x}\left(\left.\varphi_{0}\right|_{\Gamma}\right)$ in $L^{2}(\Gamma)$. However from the definition of $\eta_{\varepsilon}$ is deduced that

$$
\int_{\Gamma_{\varepsilon}^{ \pm}} \eta\left|\frac{\partial \varphi_{\varepsilon}}{\partial x}\right|^{2} \mathrm{~d} x=\varepsilon \int_{\Gamma_{\varepsilon}^{ \pm}} \eta_{\varepsilon}\left|\frac{\partial \varphi_{\varepsilon}}{\partial x}\right|^{2} \mathrm{~d} x
$$

since for $x \leq \varepsilon, \eta=x$ and $\eta_{\varepsilon}=x / \varepsilon$ (a similar result is obtained for $x \geq 1-\varepsilon$ ). Thus the second integral tends to zero since $\int_{\Gamma} \eta_{\varepsilon}\left|\frac{\partial \varphi_{\varepsilon}}{\partial x}\right|^{2} \mathrm{~d} x$ is bounded.

(2) Proof of $\int_{\Gamma_{\varepsilon}^{ \pm}} \eta_{\varepsilon}\left|\frac{\partial \varphi_{\varepsilon}}{\partial x}\right|^{2} \mathrm{~d} x \rightarrow 0$

We recall that $\varphi_{\varepsilon}$ is the unique solution in $V$ of $a_{\varepsilon}\left(\varphi_{\varepsilon}, \psi\right)=\int_{\Omega} f \bar{\psi} \mathrm{d} x \mathrm{~d} y$ for all $\psi \in V$. Taking $\psi=\tilde{e}_{\varepsilon}$ we get

$$
a_{\varepsilon}\left(\varphi_{\varepsilon}, \tilde{e}_{\varepsilon}\right)=\int_{\Omega}\left(\nabla \varphi_{\varepsilon} \cdot \nabla \overline{\tilde{e}_{\varepsilon}}-D \varphi_{\varepsilon} \overline{D \tilde{e}_{\varepsilon}}\right) \mathrm{d} x \mathrm{~d} y+\left\langle S_{ \pm} \varphi_{\varepsilon}, \tilde{e}_{\varepsilon}\right\rangle_{ \pm}+\frac{Y_{0}}{i k} \int_{\Gamma} \eta_{\varepsilon} D \varphi_{\varepsilon} \overline{D \tilde{e}_{\varepsilon}} \mathrm{d} x
$$

The last term reads more explicitely

$$
\frac{Y_{0}}{i k} \int_{\Gamma} \eta_{\varepsilon} D \varphi_{\varepsilon} \overline{D \tilde{e}_{\varepsilon}} \mathrm{d} x=\frac{Y_{0}}{i k} \int_{\Gamma} \eta_{\varepsilon}\left[M^{2} \frac{\partial \varphi_{\varepsilon}}{\partial x} \frac{\overline{\partial \tilde{e}_{\varepsilon}}}{\partial x}+i k M\left(\frac{\partial \varphi_{\varepsilon}}{\partial x} \overline{\tilde{e}_{\varepsilon}}-\varphi_{\varepsilon} \overline{\frac{\partial \tilde{e}_{\varepsilon}}{\partial x}}\right)+k^{2} \varphi_{\varepsilon} \overline{\tilde{e}_{\varepsilon}}\right] \mathrm{d} x
$$

Since $\left.\tilde{e}_{\varepsilon}\right|_{\Gamma}=\hat{v}_{\varepsilon}$ with

$$
\begin{cases}\hat{v}_{\varepsilon}(x)=0 & \text { for } \left.x \in \Gamma_{\varepsilon}=\right] \varepsilon, 1-\varepsilon[, \\ \hat{v}_{\varepsilon}(x)=\varphi_{\varepsilon}(x, h)-\varphi_{\varepsilon}\left(\varepsilon^{ \pm}, h\right) & \text { for } x \in \Gamma_{\varepsilon}^{ \pm},\end{cases}
$$

we get the simplification

$$
\frac{Y_{0}}{i k} \int_{\Gamma} \eta_{\varepsilon} D \varphi_{\varepsilon} \overline{D \tilde{e}_{\varepsilon}} \mathrm{d} x=\frac{Y_{0}}{i k} \int_{\Gamma_{\varepsilon}^{ \pm}} \eta_{\varepsilon}\left[M^{2}\left|\frac{\partial \varphi_{\varepsilon}}{\partial x}\right|^{2}+i k M\left(\frac{\partial \varphi_{\varepsilon}}{\partial x} \overline{\tilde{e}_{\varepsilon}}-\varphi_{\varepsilon} \frac{\overline{\partial \varphi_{\varepsilon}}}{\partial x}\right)+k^{2} \varphi_{\varepsilon} \overline{\tilde{e}_{\varepsilon}}\right] \mathrm{d} x
$$

Finally is deduced the upper bound

$$
\begin{gathered}
\left|\frac{Y_{0}}{k}\right| \int_{\Gamma_{\varepsilon}^{ \pm}} \eta_{\varepsilon} M^{2}\left|\frac{\partial \varphi_{\varepsilon}}{\partial x}\right|^{2} \mathrm{~d} x \leq \int_{\Omega}\left(\left|\nabla \varphi_{\varepsilon}\right|\left|\nabla \tilde{e}_{\varepsilon}\right|+\left|D \varphi_{\varepsilon}\right|\left|D \tilde{e}_{\varepsilon}\right|\right) \mathrm{d} x \mathrm{~d} y+\left|\left\langle S_{ \pm} \varphi_{\varepsilon}, \tilde{e}_{\varepsilon}\right\rangle_{ \pm}\right| \\
+\left|\frac{Y_{0}}{k}\right| \int_{\Gamma_{\varepsilon}^{ \pm}}\left[k M\left(\left|\eta_{\varepsilon}^{\frac{1}{2}} \frac{\partial \varphi_{\varepsilon}}{\partial x}\right|\left|\tilde{e}_{\varepsilon}\right|+\left|\varphi_{\varepsilon}\right|\left|\eta_{\varepsilon}^{\frac{1}{2}} \frac{\partial \varphi_{\varepsilon}}{\partial x}\right|\right)+k^{2}\left|\varphi_{\varepsilon}\right|\left|\tilde{e}_{\varepsilon}\right|\right] \mathrm{d} x+\|f\|_{L^{2}(\Omega)}\left\|\tilde{e}_{\varepsilon}\right\|_{H^{1}(\Omega)}
\end{gathered}
$$

We have used $\eta_{\varepsilon}^{\frac{1}{2}} \leq 1$. All the term in the right hand side tend to zero since

(a) $\varphi_{\varepsilon} \rightarrow \varphi_{0}$ and $\tilde{e}_{\varepsilon} \rightarrow 0$ in $H^{1}(\Omega)$;

(b) $\left.\left.\varphi_{\varepsilon}\right|_{\Gamma} \rightarrow \varphi_{0}\right|_{\Gamma}, \eta_{\varepsilon}^{\frac{1}{2}} \frac{\partial}{\partial x}\left(\left.\varphi_{\varepsilon}\right|_{\Gamma}\right) \rightarrow \frac{\partial}{\partial x}\left(\left.\varphi_{0}\right|_{\Gamma}\right)$ and $\left.\tilde{e}_{\varepsilon}\right|_{\Gamma} \rightarrow 0$ in $L^{2}(\Gamma)$;

(c) $\varphi_{\varepsilon} \rightarrow \varphi_{0}$ and $\tilde{e}_{\varepsilon} \rightarrow 0$ in $H^{\frac{1}{2}}\left(\Sigma_{ \pm}\right)$and $S_{ \pm}$is continuous from $H^{\frac{1}{2}}\left(\Sigma_{ \pm}\right)$to $H^{-\frac{1}{2}}\left(\Sigma_{ \pm}\right)$;

(d) the term $\left|\varphi_{\varepsilon}\right|\left|\eta_{\varepsilon}^{\frac{1}{2}} \frac{\partial \varphi_{\varepsilon}}{\partial x}\right|$ tends to $\left|\varphi_{0}\right|\left|\frac{\partial \varphi_{0}}{\partial x}\right|$ in $L^{1}(\Gamma)$ and $\int_{\Gamma_{\varepsilon}^{ \pm}}\left|\varphi_{0}\right|\left|\frac{\partial \varphi_{0}}{\partial x}\right| \mathrm{d} x$ tends to zero thanks to

Lebesgue's theorem.

(3) Proof of $\left\|\eta_{\varepsilon}^{\frac{1}{2}} D \tilde{e}_{\varepsilon}\right\|_{L^{2}(\Gamma)}^{2} \rightarrow 0$ 
Since $\left.\tilde{e}_{\varepsilon}\right|_{\Gamma}=\hat{v}_{\varepsilon}$ on $\Gamma$, we get

$$
\left\|\eta \eta_{\varepsilon}^{\frac{1}{2}} D \tilde{e}_{\varepsilon}\right\|_{L^{2}(\Gamma)} \leq M\left\|\eta \eta_{\varepsilon}^{\frac{1}{2}} \frac{\mathrm{d} \hat{v}_{\varepsilon}}{\mathrm{d} x}\right\|_{L^{2}(\Gamma)}+k\left\|\hat{v}_{\varepsilon}\right\|_{L^{2}(\Gamma)},
$$

and we have proved in the two previous proofs that both

$$
\left\|\eta_{\varepsilon}^{\frac{1}{2}} \frac{\mathrm{d} \hat{v}_{\varepsilon}}{\mathrm{d} x}\right\|_{L^{2}(\Gamma)}^{2}=\int_{\Gamma_{\varepsilon}^{ \pm}} \eta_{\varepsilon}\left|\frac{\partial \varphi_{\varepsilon}}{\partial x}\right|^{2} \mathrm{~d} x
$$

and $\left\|\hat{v}_{\varepsilon}\right\|_{L^{2}(\Gamma)}^{2}$ tend to zero.

Lemma B.4. If $\varphi_{\varepsilon} \in V$, the sequence solution of $\left(P_{\varepsilon}\right)$, is such that $\left\|\varphi_{\varepsilon}\right\|_{\varepsilon}$ is bounded then $\left\|\varphi_{0}-\tilde{\varphi}_{\varepsilon}\right\|_{0} \rightarrow 0$.

Proof. We note $e_{\varepsilon}=\varphi_{0}-\tilde{\varphi}_{\varepsilon} \in V_{0}$ and we will prove that

- $a_{0}\left(e_{\varepsilon}, e_{\varepsilon}\right) \rightarrow 0$,

- $e_{\varepsilon} \rightarrow 0$ in $V_{0}$.

(1) Proof of $a_{0}\left(e_{\varepsilon}, e_{\varepsilon}\right) \rightarrow 0$

For all $\psi \in V_{0}$, starting from $a_{0}\left(\varphi_{0}, \psi\right)=\int_{\Omega} f \bar{\psi} \mathrm{d} x \mathrm{~d} y=a_{\varepsilon}\left(\varphi_{\varepsilon}, \psi\right)$ we introduce the writing $a_{0}\left(\varphi_{0}, \psi\right)=$ $a_{0}\left(\varphi_{0}-\tilde{\varphi}_{\varepsilon}+\tilde{\varphi}_{\varepsilon}, \psi\right)$ to deduce

$$
a_{0}\left(\varphi_{0}-\tilde{\varphi}_{\varepsilon}, \psi\right)=a_{\varepsilon}\left(\varphi_{\varepsilon}, \psi\right)-a_{0}\left(\tilde{\varphi}_{\varepsilon}, \psi\right)
$$

Bearing in mind that $e_{\varepsilon}=\varphi_{0}-\tilde{\varphi}_{\varepsilon}$ and that $\tilde{e}_{\varepsilon}=\varphi_{\varepsilon}-\tilde{\varphi}_{\varepsilon}$ we obtain

$$
a_{0}\left(e_{\varepsilon}, \psi\right)=\int_{\Omega}\left(\nabla \tilde{e}_{\varepsilon} \cdot \nabla \bar{\psi}-D \tilde{e}_{\varepsilon} \overline{D \psi}\right) \mathrm{d} x \mathrm{~d} y+\left\langle S_{ \pm} \tilde{e}_{\varepsilon}, \psi\right\rangle_{ \pm}+\frac{Y_{0}}{i k} \int_{\Gamma}\left(\eta_{\varepsilon} D \varphi_{\varepsilon}-D \tilde{\varphi}_{\varepsilon}\right) \overline{D \psi} \mathrm{d} x .
$$

Since $\tilde{\varphi}_{\varepsilon}=\varphi_{\varepsilon}$, the last term simplifies in

$$
\frac{Y_{0}}{i k} \int_{\Gamma}\left(\eta_{\varepsilon} D \varphi_{\varepsilon}-D \tilde{\varphi}_{\varepsilon}\right) \overline{D \psi} \mathrm{d} x=\frac{Y_{0}}{i k} \int_{\Gamma_{\varepsilon}^{ \pm}}\left(\eta_{\varepsilon} M \frac{\partial \varphi_{\varepsilon}}{\partial x}-i k\left[\eta_{\varepsilon} \varphi_{\varepsilon}(x, h)-\varphi_{\varepsilon}\left(\varepsilon^{ \pm}, h\right)\right]\right) \overline{D \psi} \mathrm{d} x
$$

Now let us take $\psi=e_{\varepsilon} \in V_{0}$ to get

$$
a_{0}\left(e_{\varepsilon}, e_{\varepsilon}\right)=d_{0}\left(\tilde{e}_{\varepsilon}, e_{\varepsilon}\right)+e_{0}\left(\varphi_{\varepsilon}, e_{\varepsilon}\right)
$$

with

$$
d_{0}\left(\tilde{e}_{\varepsilon}, e_{\varepsilon}\right)=\int_{\Omega}\left(\nabla \tilde{e}_{\varepsilon} \cdot \nabla \overline{e_{\varepsilon}}-D \tilde{e}_{\varepsilon} \overline{D e_{\varepsilon}}\right) \mathrm{d} x \mathrm{~d} y+\left\langle S_{ \pm} \tilde{e}_{\varepsilon}, e_{\varepsilon}\right\rangle_{ \pm}
$$

and

$$
\begin{aligned}
e_{0}\left(\varphi_{\varepsilon}, e_{\varepsilon}\right)= & \frac{Y_{0}}{i k} \int_{\Gamma_{\varepsilon}^{ \pm}}\left(M^{2}\left(\eta_{\varepsilon} \frac{\partial \varphi_{\varepsilon}}{\partial x}\right) \overline{\frac{\partial e_{\varepsilon}}{\partial x}}+i k M\left\{\left(\eta_{\varepsilon} \frac{\partial \varphi_{\varepsilon}}{\partial x}\right) \overline{e_{\varepsilon}}-\left[\eta_{\varepsilon} \varphi_{\varepsilon}(x, h)-\varphi_{\varepsilon}\left(\varepsilon^{ \pm}, h\right)\right] \overline{\frac{\partial e_{\varepsilon}}{\partial x}}\right\}\right. \\
& \left.+k^{2}\left[\eta_{\varepsilon} \varphi_{\varepsilon}(x, h)-\varphi_{\varepsilon}\left(\varepsilon^{ \pm}, h\right)\right] \overline{e_{\varepsilon}}\right) \mathrm{d} x
\end{aligned}
$$

Using $\left.e_{\varepsilon}\right|_{\Gamma_{\varepsilon}^{ \pm}}=\left.\varphi_{0}\right|_{\Gamma_{\varepsilon}^{ \pm}}-\varphi_{\varepsilon}\left(\varepsilon^{ \pm}, h\right)$ on $\Gamma_{\varepsilon}^{ \pm}$leads to

$$
\begin{aligned}
e_{0}\left(\varphi_{\varepsilon}, e_{\varepsilon}\right)= & \frac{Y_{0}}{i k} \int_{\Gamma_{\varepsilon}^{ \pm}}\left(M^{2}\left(\eta_{\varepsilon} \frac{\partial \varphi_{\varepsilon}}{\partial x}\right) \overline{\frac{\partial \varphi_{0}}{\partial x}}\right. \\
& +i k M\left\{\left(\eta_{\varepsilon} \frac{\partial \varphi_{\varepsilon}}{\partial x}\right) \overline{\varphi_{0}(x, h)-\varphi_{\varepsilon}\left(\varepsilon^{ \pm}, h\right)}-\left[\eta_{\varepsilon} \varphi_{\varepsilon}(x, h)-\varphi_{\varepsilon}\left(\varepsilon^{ \pm}, h\right)\right] \overline{\frac{\partial \varphi_{0}}{\partial x}}\right\} \\
& \left.+k^{2}\left[\eta_{\varepsilon} \varphi_{\varepsilon}(x, h)-\varphi_{\varepsilon}\left(\varepsilon^{ \pm}, h\right)\right] \overline{\varphi_{0}(x, h)-\varphi_{\varepsilon}\left(\varepsilon^{ \pm}, h\right)}\right) \mathrm{d} x
\end{aligned}
$$


Last the modulus is found to be bounded by

$$
\begin{aligned}
\left|a_{0}\left(e_{\varepsilon}, e_{\varepsilon}\right)\right| \leq & \int_{\Omega}\left(\left|\nabla \tilde{e}_{\varepsilon}\right|\left|\nabla \overline{e_{\varepsilon}}\right|+\left|D \tilde{e}_{\varepsilon}\right|\left|D e_{\varepsilon}\right|\right) \mathrm{d} x \mathrm{~d} y+\left|\left\langle S_{ \pm} \tilde{e}_{\varepsilon}, e_{\varepsilon}\right\rangle_{ \pm}\right| \\
& +\left|\frac{Y_{0}}{k}\right| \int_{\Gamma_{\varepsilon}^{ \pm}}\left[M^{2}\left|\eta_{\varepsilon}^{\frac{1}{2}} \frac{\partial \varphi_{\varepsilon}}{\partial x}\right|\left|\frac{\partial \varphi_{0}}{\partial x}\right|\right. \\
& +k M\left(\left|\eta_{\varepsilon}^{\frac{1}{2}} \frac{\partial \varphi_{\varepsilon}}{\partial x}\right|\left|\varphi_{0}(x, h)-\varphi_{\varepsilon}\left(\varepsilon^{ \pm}, h\right)\right|+\left|\eta_{\varepsilon} \varphi_{\varepsilon}(x, h)-\varphi_{\varepsilon}\left(\varepsilon^{ \pm}, h\right)\right|\left|\frac{\partial \varphi_{0}}{\partial x}\right|\right), \\
& \left.+k^{2}\left|\eta_{\varepsilon} \varphi_{\varepsilon}(x, h)-\varphi_{\varepsilon}\left(\varepsilon^{ \pm}, h\right)\right|\left|\varphi_{0}(x, h)-\varphi_{\varepsilon}\left(\varepsilon^{ \pm}, h\right)\right|\right] \mathrm{d} x .
\end{aligned}
$$

All the terms in the right hand side tend to zero (and thus $\left|a_{0}\left(e_{\varepsilon}, e_{\varepsilon}\right)\right| \rightarrow 0$ ) because

(a) $\tilde{e}_{\varepsilon} \rightarrow 0$ and $e_{\varepsilon} \rightarrow 0$ in $H^{1}(\Omega)$

(b) $\int_{\Gamma_{\varepsilon}^{ \pm}}\left|\eta_{\varepsilon} \varphi_{\varepsilon}(x, h)-\varphi_{\varepsilon}\left(\varepsilon^{ \pm}, h\right)\right|^{2} \mathrm{~d} x \leq 2\left(\varepsilon\left|\varphi_{\varepsilon}\left(\varepsilon^{ \pm}, h\right)\right|^{2}+\int_{\Gamma_{\varepsilon}^{ \pm}}\left|\varphi_{\varepsilon}(x, h)\right|^{2} \mathrm{~d} x\right) \rightarrow 0$

(c) $\int_{\Gamma_{\varepsilon}^{ \pm}}\left|\varphi_{0}(x, h)-\varphi_{\varepsilon}\left(\varepsilon^{ \pm}, h\right)\right|^{2} \mathrm{~d} x \leq 2\left(\int_{\Gamma_{\varepsilon}^{ \pm}}\left|\varphi_{0}(x, h)\right|^{2} \mathrm{~d} x+\varepsilon\left|\varphi_{\varepsilon}\left(\varepsilon^{ \pm}, h\right)\right|^{2}\right) \rightarrow 0$

(d) $\tilde{e}_{\varepsilon} \rightarrow 0$ and $e_{\varepsilon} \rightarrow 0$ in $H^{\frac{1}{2}}\left(\Sigma_{ \pm}\right)$,

(e) $\eta_{\varepsilon}^{\frac{1}{2}} \frac{\partial}{\partial x}\left(\left.\varphi_{\varepsilon}\right|_{\Gamma}\right) \rightarrow \frac{\partial}{\partial x}\left(\left.\varphi_{0}\right|_{\Gamma}\right)$ in $L^{2}(\Gamma)$

To prove the first point, we have used the fact that $\varphi_{\varepsilon} \rightarrow \varphi_{0}$ in $H^{1}(\Omega)$ and also that since $\left\|\varphi_{\varepsilon}-\tilde{\varphi}_{\varepsilon}\right\|_{\varepsilon} \rightarrow 0$ we get $\tilde{\varphi}_{\varepsilon} \rightarrow \varphi_{\varepsilon}$ in $H^{1}(\Omega)$. We deduce that $\tilde{\varphi}_{\varepsilon} \rightarrow \varphi_{0}$ in $H^{1}(\Omega)$ which means that $e_{\varepsilon} \rightarrow 0$ in $H^{1}(\Omega)$.

(2) Proof of $e_{\varepsilon} \rightarrow 0$ in $V_{0}$

We split $a_{0}(\varphi, \psi)$ in a coercive part and a compact perturbation part

$$
a_{0}(\varphi, \psi)=b_{0}(\varphi, \psi)+c_{0}(\varphi, \psi)
$$

where

$$
b_{0}(\varphi, \psi)=\int_{\Omega}\left[\left(1-M^{2}\right) \frac{\partial \varphi}{\partial x} \frac{\overline{\partial \psi}}{\partial x}+\frac{\partial \varphi}{\partial y} \frac{\overline{\partial \psi}}{\partial y}+\varphi \bar{\psi}\right] \mathrm{d} x \mathrm{~d} y+\left\langle S_{ \pm}^{e} \varphi, \psi\right\rangle_{ \pm}+\frac{Y}{i k} \int_{\Gamma} D \varphi \overline{D \psi} \mathrm{d} x
$$

and

$$
c_{0}(\varphi, \psi)=\int_{\Omega}\left[-\left(1+k^{2}\right) \varphi \bar{\psi}+i k M\left(\varphi \frac{\overline{\partial \psi}}{\partial x}-\frac{\partial \varphi}{\partial x} \bar{\psi}\right)\right] \mathrm{d} x \mathrm{~d} y+\left\langle S_{ \pm}^{p} \varphi, \psi\right\rangle_{ \pm} .
$$

Since $c_{0}(\varphi, \psi)$ is compact, $e_{\varepsilon} \rightarrow 0$ in $H^{1}(\Omega)$ implies that $c_{0}\left(e_{\varepsilon}, e_{\varepsilon}\right) \rightarrow 0$ and the coercivity of $b_{0}(\varphi, \psi)$ on $V_{0}$ leads to $\left\|e_{\varepsilon}\right\|_{0}=\left\|\varphi_{0}-\tilde{\varphi}_{\varepsilon}\right\|_{0} \rightarrow 0$.

\section{Appendix C. Exact RADiation CONDITION AND DTN operator}

In order to formulate the diffraction problem, we need to introduce the so-called modes of the duct (without a liner) which are the solutions with separated variables of $-\Delta \varphi+D^{2} \varphi=0$ in $\Omega$ with $\partial \varphi / \partial y=0$ at $y=0$ and $y=h$. They are well-known and are given by

$$
u_{n}^{ \pm}=\mathrm{e}^{i \beta_{n}^{ \pm} x} \cos \left(\frac{n \pi y}{h}\right),
$$

where $\beta_{n}^{ \pm}$is given by 
- If $n \leq N=k h / \pi \sqrt{1-M^{2}}$

$$
\beta_{n}^{ \pm}=\frac{\left[-k M \pm \sqrt{k^{2}-\frac{n^{2} \pi^{2}}{h^{2}}\left(1-M^{2}\right)}\right]}{1-M^{2}} .
$$

In that case, $\beta_{n}^{ \pm}$is real and $u_{n}^{ \pm}$is a propagative mode. The + modes correspond to a positive group velocity and thus propagate downstream, whereas the - modes propagate upstream.

- If $n>N$ :

$$
\beta_{n}^{ \pm}=\frac{\left[-k M \pm i \sqrt{\frac{n^{2} \pi^{2}}{h^{2}}\left(1-M^{2}\right)-k^{2}}\right]}{1-M^{2}} .
$$

In that case, $u_{n}^{ \pm}$is an evanescent mode which oscillates.

To derive problem (3.1) set in the bounded domain $\Omega=\{(x, y) \in \Omega ;-R<x<R\}$, we need to define on the artificial boundaries $\Sigma_{ \pm}=\{(x, y) ; x= \pm R$ and $0<y<h\}$ some suitable boundary conditions. They are deduced from the modal decomposition of $u$ in the exterior domains

$$
\Omega_{ \pm}=\{(x, y) \in \Omega ; \pm x>R\},
$$

which reads $u=\sum_{n>N}\left(u, w_{n}\right)_{\Sigma_{ \pm}} \mathrm{e}^{\mathrm{i} \beta_{n}^{ \pm}(x \mp R)} w_{n}(y)$ in $\Omega_{ \pm}$where $w_{n}(y)=\sqrt{\frac{2}{h}} \cos \left(\frac{n \pi y}{h}\right)$ for $n>0, w_{0}(y)=\sqrt{\frac{1}{h}}$ and $(u, v)_{\Sigma^{ \pm}}=\int_{\Sigma^{ \pm}} u \bar{v} \mathrm{~d} y$. The deduced exact boundary conditions is

$$
\frac{\partial u}{\partial n}=-T_{ \pm} u, \text { on } \Sigma_{ \pm}
$$

where the Dirichet-to-Neumann operators are defined as follows

$$
\begin{aligned}
T_{ \pm}: H^{\frac{1}{2}}\left(\Sigma_{ \pm}\right) & \rightarrow H^{-\frac{1}{2}}\left(\Sigma_{ \pm}\right) \\
u & \rightarrow \mp i \sum_{n \geq 0} \beta_{n}^{ \pm}\left(u, w_{n}\right)_{\Sigma^{ \pm}} w_{n}
\end{aligned}
$$

Acknowledgements. The authors gratefully acknowledge the Agence Nationale de la Recherche (AEROSON project, ANR-09-BLAN-0068-02 program) for financial support.

\section{REFERENCES}

[1] K. Ingard, Influence of Fluid Motion Past a Plane Boundary on Sound Reflection, Absorption, and Transmission. J. Acoust. Soc. Am. 31 (1959) 1035-1036.

[2] M. Myers, On the acoustic boundary condition in the presence of flow. J. Acoust. Soc. Am. 71 (1980) 429-434.

[3] W. Eversman and R.J. Beckemeyer, Transmission of Sound in Ducts with Thin Shear layers-Convergence to the Uniform Flow Case. J. Acoust. Soc. Am. 52 (1972) 216-220.

[4] B.J. tester, Some Aspects of "Sound" Attenuation in Lined Ducts containing Inviscid Mean Flows with Boundary Layers. J. Sound Vib. 28 (1973) 217-245

[5] G. Gabard and R.J. Astley, A computational mode-matching approach for sound propagation in three-dimensional ducts with flow. J. Acoust. Soc. Am. 315 (2008) 1103-1124.

[6] G. Gabard, Mode-Matching Techniques for Sound Propagation in Lined Ducts with Flow. Proc. of the 16th AIAA/CEAS Aeroacoustics Conference.

[7] R. Kirby, A comparison between analytic and numerical methods for modeling automotive dissipative silencers with mean flow. J. Acoust. Soc. Am. 325 (2009) 565-582

[8] R. Kirby and F.D. Denia, Analytic mode matching for a circular dissipative silencer containing mean flow and a perforated pipe. J. Acoust. Soc. Am. 122 (2007) 71-82. 
[9] Y. Aurégan and M. Leroux, Failures in the discrete models for flow duct with perforations: an experimental investigation. J. Acoust. Soc. Am. 265 (2003) 109-121

[10] E.J. Brambley, Low-frequency acoustic reflection at a hardsoft lining transition in a cylindrical duct with uniform flow. J. Engng. Math. 65 (2009) 345-354.

[11] S. Rienstra and N. Peake, Modal Scattering at an Impedance Transition in a Lined Flow Duct. Proc. of 11th AIAA/CEAS Aeroacoustics Conference, Monterey, CA, USA (2005).

[12] S.W. Rienstra, Acoustic Scattering at a Hard-Soft Lining Transition in a Flow Duct. J. Engrg. Math. 59 (2007) $451-475$.

[13] S. Rienstra, A classification of duct modes based on surface waves. Wave Motion 37 (2003) 119-135.

[14] E.J. Brambley and N. Peake, Surface-waves, stability, and scattering for a lined duct with flow. Proc. of AIAA Paper (2006) 2006-2688.

[15] B.J. tester, The Propagation and Attenuation of sound in Lined Ducts containing Uniform or "Plug" Flow. J. Acoust. Soc. Am. 28 (1973) 151-203

[16] P.G. Daniels, On the Unsteady Kutta Condition. Quarterly J. Mech. Appl. Math. 31 (1985) 49-75.

[17] D.G. Crighton, The Kutta condition in unsteady flow. Ann. Rev. Fluid Mech. 17 (1985) 411-445.

[18] M. Brandes and D. Ronneberger, Sound amplification in flow ducts lined with a periodic sequence of resonators. Proc. of AIAA paper, 1st AIAA/CEAS Aeroacoustics Conference, Munich, Germany (1995) 95-126.

[19] Y. Aurégan, M. Leroux and V. Pagneux, Abnormal behaviour of an acoustical liner with flow. Forum Acusticum, Budapest (2005).

[20] B. Regan and J. Eaton, Modeling the influence of acoustic liner non-uniformities on duct modes. J. Acoust. Soc. Am. 219 (1999) 859-879.

[21] K.S. Peat and K.L. Rathi, A Finite Element Analysis of the Convected Acoustic Wave Motion in Dissipative Silencers. J. Acoust. Soc. Am. 184 (1995) 529-545.

[22] W. Eversman, The Boundary condition at an Impedance Wall in a Non-Uniform Duct with Potential Mean Flow. J. Acoust. Soc. Am. 246 (2001) 63-69.

[23] S.N. Chandler-Wilde and J. Elschner, Variational Approach in Weighted Sobolev Spaces to Scattering by Unbounded Rough Surfaces. SIAM J. Math. Anal. SIMA 42 (2010) 2554-2580.

[24] B. Guo and C. Schwab, Analytic regularity of Stokes flow on polygonal domains in countably weighted Sobolev spaces. J. Comput. Appl. Math. 190 (2006) 487-519.

[25] M. Dambrine and G. Vial, A multiscale correction method for local singular perturbations of the boundary. ESAIM: M2AN 41 (2007) 111-127.

[26] P. Ciarlet and S. Kaddouri, Multiscaled asymptotic expansions for the electric potential: surface charge densities and electric fields at rounded corners. Math. Models Methods Appl. Sci. 17 (2007) 845-876.

[27] S. Tordeux, G. Vial and M. Dauge, Matching and multiscale expansions for a model singular perturbation problem. C. R. Acad. Sci. Paris Ser. I 343 (2006) 637-642.

[28] M. Costabel, M. Dauge and M. Surib, Numerical Approximation of a Singularly Perturbed Contact Problem. Computer Methods Appl. Mech. Engrg. 157 (1998) 349-363.

[29] A.-S. Bonnet-Ben Dhia, L. Dahi, E. Lunéville and V. Pagneux, Acoustic diffraction by a plate in a uniform flow. Math. Models Methods Appl. Sci. 12 (2002) 625-647.

[30] D. Martin, Code éléments finis MELINA. Available at http://anum-maths.univ-rennes1.fr/melina/danielmartin/melina/ www/somm_html/fr-main.html

[31] S. Job, E. Lunéville and J.-F. Mercier, Diffraction of an acoustic wave in a uniform flow: a numerical approach. J. Comput. Acoust. 13 (2005) 689-709. 